\title{
Continuous Adjoint Approach for the Spalart-Allmaras Model in Aerodynamic Optimization
}

\author{
Alfonso Bueno-Orovio* \\ University of Oxford, Oxford, England OX1 3QD, United Kingdom \\ Carlos Castro $\ddagger$ \\ Universidad Politécnica de Madrid, 28040 Madrid, Spain \\ Francisco Palacios \\ Stanford University, Stanford, California 94305 \\ and \\ Enrique Zuazua $\stackrel{\S}{\underline{s}}$ \\ Basque Center for Applied Mathematics, 48160 Derio, Spain
}

DOI: $10.2514 / 1 . J 051307$

\begin{abstract}
In this paper, the continuous adjoint method to compute shape sensitivities in aerodynamic design with turbulence modeling is described and developed. The focus is on compressible flows described by the Reynolds-averaged Navier-Stokes equations and the classical Spalart-Allmaras model for turbulence. Turbulence modeling usually requires, in particular, computation of the distance to the surface. Here, this distance is incorporated to the system as a new variable, solving the Eikonal equation. The accuracy of the sensitivity derivatives obtained with the complete turbulent approach is assessed by comparison with finite difference computations and the classical continuous adjoint with frozen viscosity, showing substantial improvements in the convergence properties of the method and in the quality of the obtained gradients. The validity of the overall methodology is illustrated with several design examples, including the optimization of three-dimensional geometries in combination with advanced freeform techniques for mesh deformation.
\end{abstract}

\begin{tabular}{|c|c|c|}
\hline \multicolumn{3}{|r|}{ Nomenclature } \\
\hline$A_{d}^{d}$ & $=$ & $\begin{array}{l}\text { adjoint operator of the Eikonal equation with } \\
\text { respect to distance to surface }\end{array}$ \\
\hline$A_{U}^{U}$ & $=$ & $\begin{array}{l}\text { adjoint operator of the flow equation with } \\
\text { respect to flow variables }\end{array}$ \\
\hline$A_{U}^{\hat{v}}$ & $=$ & $\begin{array}{l}\text { adjoint operator of the flow equation with } \\
\text { respect to the turbulent variable }\end{array}$ \\
\hline$A_{\hat{v}}^{d}$ & $=$ & $\begin{array}{l}\text { adjoint operator of the turbulent equation with } \\
\text { respect to distance to surface }\end{array}$ \\
\hline$A_{\hat{v}}^{U}$ & $=$ & $\begin{array}{l}\text { adjoint operator of the turbulent equation with } \\
\text { respect to flow variables }\end{array}$ \\
\hline$A_{\hat{v}}^{\hat{v}}$ & & $\begin{array}{l}\text { adjoint operator of the turbulent equation with } \\
\text { respect to turbulent variable }\end{array}$ \\
\hline$B_{S}$ & $=$ & boundary terms of adjoint equation \\
\hline$B^{i}$ & $=$ & Bernstein polynomial of order $i$ \\
\hline$c_{d}$ & $=$ & drag coefficient \\
\hline$c_{l}$ & $=$ & lift coefficient \\
\hline$C_{p}$ & $=$ & specific heat at constant pressure \\
\hline$c_{p}$ & $=$ & pressure coefficient \\
\hline$d_{S}$ & $=$ & distance to boundary of obstacle $S$ \\
\hline$E$ & $=$ & flow energy \\
\hline f & $=$ & $P \mathbf{n}-\bar{\sigma} \cdot \mathbf{n} ;$ inviscid and viscous forces \\
\hline
\end{tabular}

Presented as Paper 2011-1299 at the AIAA Aerospace Sciences Meeting including the New Horizons Forum and Aerospace Exposition, Orlando, FL, 4-7 January 2011; received 23 March 2011; revision received 1 September 2011; accepted for publication 2 September 2011. Copyright $\odot 2011$ by the American Institute of Aeronautics and Astronautics, Inc. All rights reserved. Copies of this paper may be made for personal or internal use, on condition that the copier pay the $\$ 10.00$ per-copy fee to the Copyright Clearance Center, Inc., 222 Rosewood Drive, Danvers, MA 01923; include the code 0001-1452/ 12 and $\$ 10.00$ in correspondence with the CCC.

*Research Assistant, Department of Computer Science.

'Professor, Department of Mathematics and Information, School of Civil Engineers, Canales y Puertos.

Engineering Research Associate, Department of Aeronautics and Astronautics. Member AIAA.

${ }^{\S}$ Scientific Director; Ikerbasque Research Professor, Ikerbasque, Basque Foundation for Science, 48011 Bilbao, 48011.

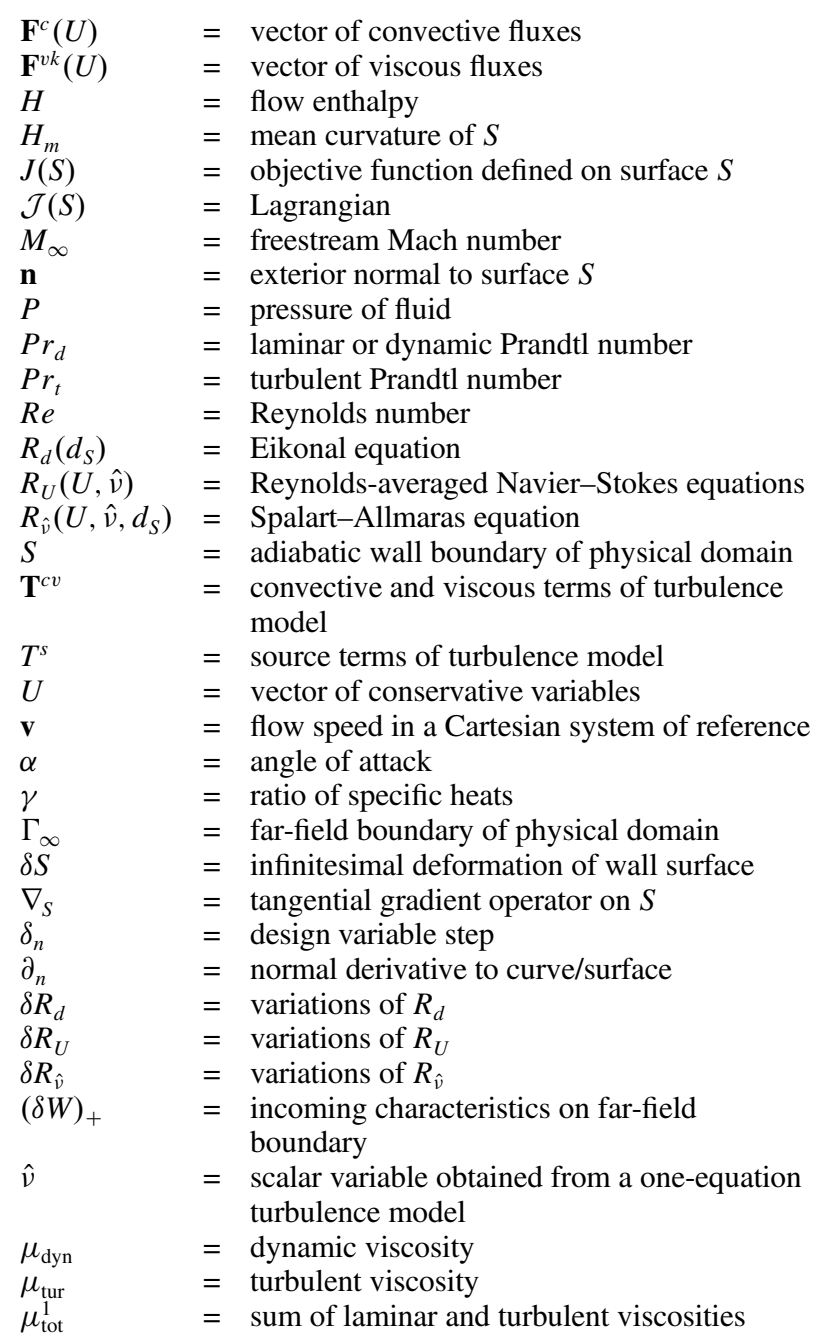




$\begin{array}{lll}\mu_{\mathrm{tot}}^{2} & = & \text { sum of laminar and turbulent viscosities divided } \\ & = & \text { by corresponding Prandtl number } \\ \rho & =\text { flow density } \\ \bar{\Sigma}^{\varphi} & = & \text { adjoint stress tensor } \\ \bar{\sigma} & = & \text { adjoint velocity vector } \\ \varphi & = & \text { vector of Lagrange multipliers of flow equations } \\ \Psi_{U} & = & \text { Lagrange multiplier of the Eikonal equation } \\ \psi_{d} & = & \text { Lagrange multiplier of the turbulent variable } \\ \psi_{\hat{v}} & = & \text { physical domain }\end{array}$

\section{Introduction}

$\mathbf{T}$ HIS paper contains a complete formulation of the continuous adjoint approach for the shape optimization of an obstacle with a boundary $S$ immersed in a fluid governed by the Reynoldsaveraged Navier-Stokes (RANS) equations. We focus on the Spalart-Allmaras turbulence model [1] , due to its wide use in aerodynamic industry. As a result, a system of partial differential equations (PDEs), suitable for numerical simulation at a relatively low cost but still retaining significant properties of the turbulent flow, is obtained.

Shape optimization methods have grown in importance in aerodynamic design within the last decade. In gradient-based optimization techniques, the goal is to minimize a suitable cost or objective function (drag coefficient, deviation from a prescribed surface pressure distribution, etc.) with respect to a set of design variables (defining, for example, an airfoil profile or aircraft surface). Minimization is achieved by means of an iterative process that requires the computation of the gradient or sensitivity derivative of the cost function with respect to the design variables.

Gradients can be computed in a variety of ways, the most actively pursued one being the adjoint method [2-5] due, among other factors, to its ability of computing these derivatives at a cost comparable with that of numerically solving the state PDEs. Adjoint methods are conventionally subdivided into continuous and discrete methods. In the continuous approach, the adjoint equations are derived from the governing PDEs and subsequently discretized to obtain a descent direction for the discrete cost functional, whereas in the discrete approach, this descent direction is directly obtained from the adjoint equations of the discretized PDEs [6].

In the continuous adjoint approach, the adjoint system is a linear system of PDEs that can be discretized using any convergent numerical scheme. One can take advantage of this, but it requires a suitable numerical analysis of the underlying system of equations. Note that the adjoint equations are not derived directly from a physical model, and their numerical analysis is not similar to the one required for the fluid equations. In particular, stability and convergence must be analyzed independently. It is worth mentioning that this is not an easy problem when singularities appear. For instance, consistency of adjoint problems in the presence of shock waves is a difficult topic [7-10]. Moreover, mathematical and physical understanding of the adjoint problem is aided by considering the continuous approach, as exemplified by analytic adjoint solutions for one-dimensional Euler problems [11].

On the contrary, the discrete adjoint provides the exact gradient of the discretized objective function (as the finite differences method or the complex step method [12]). The discrete adjoint is also often considerably harder than the continuous one in terms of operation counts and memory requirements, although some efficient codes have been developed to mitigate these limitations [13-15]. Besides, the discrete adjoint becomes complex for higher-order schemes (see [6] and references therein), and it is not always well justified since it requires the linearization of the underlying numerical scheme used to approximate the flow system. Any efficient second-order numerical scheme able to approximate the conservative part of the fluid system in the presence of singular solutions is nondifferentiable in nature. Thus, any approximation of the linearized discrete system should be carefully analyzed from the mathematical point of view. Finally, related with the discrete adjoint, it is interesting to highlight the concept of dual consistency [16], which leads to a discrete adjoint problem that is a consistent discretization of the continuous adjoint problem that we are going to derive in this paper.

To the best of the authors' knowledge, the only previous contribution concerning such a continuous adjoint approach to the RANS equations in conjunction with a turbulence model is the work of Zymaris et al. [17]. Their work, however, is devoted to the incompressible version of the Navier-Stokes equations, and it is restricted to interior flows in duct geometries. Furthermore, only sensitivities regarding the total pressure loss functional in the duct are considered. Some other studies have also considered variations in turbulent viscosity, although they are all based on the discrete adjoint approach: Nielsen et al. [18], Dwight and Brezillon [19], Anderson and Bonhaus [20], Lee and Kim [21], Mavriplis [22], and Kim et al. [23]. We refer to the introduction of Zymaris et al. [17] for a more detailed description of the previous literature on this subject. Compared with the previously mentioned works, the present paper develops the continuous adjoint method rather than the discrete one. Also note that the present paper deals with the compressible steadystate flow equations, with applications for external flows in aerodynamic design.

From a practical point of view, a nontrivial question rises when computing the distance to the surface, usually required by the turbulence model. In this work, we solve this problem by characterizing this distance as a solution of the Eikonal equation. Thus, the final system of equations incorporates the Eikonal equation, taking part of the continuous formulation of the problem.

The dependence of turbulence models with respect to the distance to the surface is an additional difficulty in RANS aerodynamic optimization problems. The very small deformations required to approximate the gradient by finite differences might not be properly seen by either an Eikonal or even a brute-force distance solver. Moreover, some recent works [24,25] have posed some serious concerns on the use of discrete methods in control problems associated to Eikonal- and Hamilton-Jacobi-related problems. On the contrary, our continuous formulation incorporates in a natural way the dependence of the turbulence model with respect to the distance to the surface by means of the linearization of the Eikonal equation, thus also opening the door to consider functionals of interest with an explicit dependence on this distance by means of domain integrals.

The organization of the paper is as follows. In Sec. II, we describe the model and state the optimization problem. In Sec. III, we derive the continuous adjoint method to compute surface sensitivities of the RANS equations. This derivation is done in a general framework, totally independent of the choice of turbulence model, and particularized for the Spalart-Allmaras one. The practical implementation of the method is then described in Sec. IV. Some numerical experiments illustrating the relevance of the developments described in this work are presented in Sec. V. For purposes of clarity, equations of the models and their linearization, as well as details on the calculations of the surface sensitivity, are given in the appendices at the end of this work.

\section{Description of the Problem}

The Navier-Stokes equations $[\underline{26}, \underline{27}]$ (see Appendix A) describe the conservation of mass, momentum, and energy in a viscous fluid. Classical aeronautical applications assume that the air is governed by these Navier-Stokes equations on a domain $\Omega \subset \mathbb{R}^{3}$ delimited by disconnected boundaries divided into a far field $\Gamma_{\infty}$ and adiabatic walls that we denote by $S$. Their steady-state formulation (without source terms) can be written in the following form:

$$
\begin{cases}R_{U}(U, \hat{v})=\nabla \cdot \mathbf{F}^{c}-\nabla \cdot\left(\mu_{\mathrm{tot}}^{1} \mathbf{F}^{v 1}+\mu_{\mathrm{tot}}^{2} \mathbf{F}^{v 2}\right)=0 & \text { in } \Omega \\ \mathbf{v}=0 & \text { on } S \\ \partial_{n} T=0 & \text { on } S \\ (W)_{+}=W_{\infty} & \text { on } \Gamma_{\infty}\end{cases}
$$

where $U=\left(\rho, \rho v_{1}, \rho v_{2}, \rho v_{3}, \rho E\right)^{\top}$ stands for the vector of conservative variables, $\rho$ is the density, $E$ is the energy, $\mathbf{v}=$ $\left(v_{1}, v_{2}, v_{3}\right) \in \mathbb{R}^{3}$ is the flow speed in a Cartesian system of reference, 
and $T$ is the temperature. The last equation in Eq. (1) represents classical far-field boundary conditions simulating the fluid behavior at infinity. The vectors $\mathbf{F}^{c}(U)=\left(\mathbf{F}_{1}^{c}, \mathbf{F}_{2}^{c}, \ldots, \mathbf{F}_{5}^{c}\right)^{\top}$ are the convective fluxes and $\mathbf{F}^{v k}(U)=\left(\mathbf{F}_{1}^{v k}, \mathbf{F}_{2}^{v k}, \ldots, \mathbf{F}_{5}^{v k}\right)^{\top}, k=1,2$, are the viscous fluxes, where we have considered separately the contribution of the viscous forces and the heat flux transfer. Here, $(\cdot)^{\top}$ denotes transposition.

As usual in turbulence modeling based upon the Boussinesq hypothesis, which states that the effect of turbulence can be represented as an increased viscosity, the viscosity is divided into laminar $\mu_{\mathrm{dyn}}$ and turbulent $\mu_{\mathrm{tur}}$ components. The laminar or dynamic viscosity is usually taken to be only dependent on the temperature $\mu_{\mathrm{dyn}}=\mu_{\mathrm{dyn}}(T)$, whereas $\mu_{\mathrm{tur}}$ is obtained from a suitable turbulence model involving the flow and a set of new variables $\hat{v}$, i.e., $\mu_{\text {tur }}=\mu_{\text {tur }}(U, \hat{v})$. Here, we assume that $\hat{v}$ is a single scalar variable obtained from a one-equation turbulence model, which in general form can be written as

$$
\begin{cases}R_{\hat{v}}\left(U, \hat{v}, d_{S}\right)=\nabla \cdot \mathbf{T}^{c v}-T^{s}=0 & \text { in } \Omega \\ \hat{v}=0 & \text { on } S \\ \hat{v}_{\infty}=\sigma_{\infty} \nu_{\infty} & \text { on } \Gamma_{\infty}\end{cases}
$$

where $\mathbf{T}^{c v}=\mathbf{T}^{c v}\left(U, \hat{v}, d_{S}\right)$ stands for the convective and viscous terms, $T^{s}=T^{s}\left(U, \hat{v}, d_{S}\right)$ represents the source term, and $d_{S}$ is the distance to the boundary of the obstacle $S$. In the particular case of the Spalart-Allmaras model, the convective, viscous, and source terms are given in Appendix B. The far-field boundary condition for the turbulent viscosity in Eq. (2) imposes some fraction of the laminar viscosity at the far field. This is usual in turbulence modeling [1], where $\sigma_{\infty}$ is a turbulence model constant, usually ranging between 3 and 5 . On viscous walls, $\hat{v}$ is set to zero, corresponding to the absence of turbulent eddies very near to the wall.

Turbulence and mainstream flow then become coupled by replacing the dynamic viscosity in the momentum and energy equations in the Navier-Stokes equations with

$$
\mu_{\mathrm{tot}}^{1}=\mu_{\mathrm{dyn}}+\mu_{\mathrm{tur}}, \quad \mu_{\mathrm{tot}}^{2}=\frac{\mu_{\mathrm{dyn}}}{P r_{d}}+\frac{\mu_{\mathrm{tur}}}{P r_{t}}
$$

where $\operatorname{Pr}_{d}$ and $\operatorname{Pr}_{t}$ are, respectively, the dynamic and turbulent Prandtl numbers. Here, $\mu_{\text {tot }}^{2}$ represents the effective thermal conductivity that we write in this nonstandard notation to obtain reduced expressions in the calculus below.

Note that, in Eq. (2), we have incorporated the distance variable $d_{S}$, which is common in turbulence modeling and, in particular, it appears in the considered Spalart-Allmaras model. The new variable $d_{S}(S)$ solves the so-called Eikonal equation:

$$
\begin{cases}R_{d}\left(d_{S}\right)=\left|\nabla d_{S}\right|^{2}-1=0 & \text { in } \Omega \\ d_{S}=0 & \text { on } S\end{cases}
$$

Systems (1), (2), and (4), together with a suitable equation of state to describe the fluid thermodynamics, constitute a complete system of equations and boundary conditions for the flow variables [28,29].

A key element for the definition of an optimal shape design problem is the objective function. In this case, we introduce an objective function that is assumed to be only dependent on the values of the flow variables at the boundary $S$. As shown in [5], for the Navier-Stokes system, only objective functions depending on $\mathbf{f}$ and the temperature $T$ are allowed for continuous adjoint optimization, with $\mathbf{f}$ given by

$$
\mathbf{f}=\left(f_{1}, f_{2}, f_{3}\right)=P \mathbf{n}-\bar{\sigma} \cdot \mathbf{n}, \quad \bar{\sigma}=\mu_{\mathrm{tot}}^{1} \bar{\tau}
$$

where $\mathbf{n}$ denotes the exterior normal to the surface $S, P$ is the pressure of the fluid, and $\bar{\sigma}$ is the second-order tensor of viscous stresses, with $\bar{\tau}$ given in Appendix A. Note that this includes, in particular, functionals depending only on the pressure $P$, since it can be written as a function of $\mathbf{f}$, using the fact that $\mathbf{n} \cdot \bar{\sigma} \cdot \mathbf{n}=0$ on the boundary [్]. More precisely,

$$
P=\mathbf{n} \cdot(P \mathbf{n}-\bar{\sigma} \cdot \mathbf{n})=\mathbf{f} \cdot \mathbf{n} \quad \text { on } S
$$

In the presence of turbulence modeling, a similar situation applies, the only difference being that we can now add a dependence on the unknown turbulence variable $\partial_{n} \hat{v}$ on $S$. Thus, for the purposes of the present study, we will consider the following general choice of objective function:

$$
J(S)=\int_{S} j\left(\mathbf{f}, T, \partial_{n} \hat{v}, \mathbf{n}\right) \mathrm{d} s
$$

\section{Variation of the Objective Function: The Adjoint Approach}

As usual in the adjoint approach, flow equations are incorporated to the cost functional as constraints by means of a Lagrange multiplier for each equation, $\Psi_{U}^{\top}=\left(\psi_{1}, \psi_{2}, \psi_{3}, \psi_{4}, \psi_{5}\right), \psi_{\hat{v}}$, and $\psi_{d}$. In this way, the Lagrangian reads

$$
\begin{aligned}
& \mathcal{J}(S)=\int_{S} j\left(\mathbf{f}, T, \partial_{n} \hat{v}, \mathbf{n}\right) \mathrm{d} s+\int_{\Omega}\left(\Psi_{U}^{\top} R_{U}(U, \hat{v})\right. \\
& \left.\quad+\psi_{\hat{v}} R_{\hat{v}}\left(U, \hat{v}, d_{S}\right)+\psi_{d} R_{d}\left(d_{S}\right)\right) \mathrm{d} \Omega
\end{aligned}
$$

Let us consider an arbitrary (but small) perturbation of the boundary $S$ that, without loss of generality, can be parameterized by an infinitesimal deformation of size $\delta S$ along the normal direction to the surface $S$. The new surface obtained after the deformation is then given by

$$
S^{\prime}=\{\mathbf{x}+\delta S \mathbf{n}, \mathbf{x} \in S\}
$$

where for small deformations, the following holds [30]:

$$
\left\{\begin{array}{l}
\delta \mathbf{n}=-\nabla_{S}(\delta S) \\
\delta(d s)=-2 H_{m} \delta S d s
\end{array}\right.
$$

where $H_{m}$ is the mean curvature of $S$ computed as $\left(\kappa_{1}+\kappa_{2}\right) / 2$, and $\left(\kappa_{1}, \kappa_{2}\right)$ are curvatures in two orthogonal directions on the surface. Here, $\nabla_{S}$ represents the tangential gradient operator on $S$. Note that $\nabla_{S}(\delta S)$ is a tangent vector to $S$ that we write as a vector in $\mathbb{R}^{3}$ with a null component normal to $S$.

Assuming a regular flow solution $U$ and a smooth boundary $S$, the variation of the functional $J$ due to the deformation can be evaluated as

$$
\begin{aligned}
\delta \mathcal{J} & =\int_{S} \delta j\left(\mathbf{f}, T, \partial_{n} \hat{v}, \mathbf{n}\right) \mathrm{d} s+\int_{\delta S} j\left(\mathbf{f}, T, \partial_{n} \hat{v}, \mathbf{n}\right) \mathrm{d} s \\
& +\int_{\Omega}\left(\Psi_{U}^{\top} \delta R_{U}(U, \hat{v})+\psi_{\hat{v}} \delta R_{\hat{v}}\left(U, \hat{v}, d_{S}\right)+\psi_{d} \delta R_{d}\left(d_{S}\right)\right) \mathrm{d} \Omega
\end{aligned}
$$

where $\delta R_{U}, \delta R_{\hat{v}}$, and $\delta R_{d}$ represent the variations of $R_{U}, R_{\hat{v}}$, and $R_{d}$, respectively. Using the convention of summation of repeated indexes, $i=1,2,3$, the two first terms in the previous equation read

$$
\begin{aligned}
\delta j\left(\mathbf{f}, T, \partial_{n} \hat{v}, \mathbf{n}\right)=\frac{\partial j}{\partial f_{i}} \delta f_{i}+\frac{\partial j}{\partial T} \delta T+\frac{\partial j}{\partial\left(\partial_{n} \hat{v}\right)} \delta\left(\partial_{n} \hat{v}\right)-\frac{\partial j}{\partial \mathbf{n}} \cdot \nabla_{S}(\delta S) \\
=\frac{\partial j}{\partial \mathbf{f}} \cdot(\delta P \mathbf{n}-\delta \bar{\sigma} \cdot \mathbf{n})+\frac{\partial j}{\partial T} \delta T+\frac{\partial j}{\partial\left(\partial_{n} \hat{v}\right)} \delta\left(\partial_{n} \hat{v}\right) \\
-\left(\frac{\partial j}{\partial \mathbf{n}}+\frac{\partial j}{\partial \mathbf{f}} P-\frac{\partial j}{\partial \mathbf{f}} \cdot \bar{\sigma}\right) \cdot \nabla_{S}(\delta S)
\end{aligned}
$$$$
\int_{\delta S} j\left(\mathbf{f}, T, \partial_{n} \hat{v}, \mathbf{n}\right) \mathrm{d} s=\int_{S}\left(\frac{\partial j}{\partial f_{i}} \partial_{n} f_{i}+\frac{\partial j}{\partial T} \partial_{n} T\right.
$$$$
\left.+\frac{\partial j}{\partial\left(\partial_{n} \hat{v}\right)} \partial_{n}^{2} \hat{v}-2 H_{m} j\right) \delta S \mathrm{~d} s
$$ 
Note that, in Eq. (12), we have written the variation $\delta \mathbf{f}$ in terms of $\delta P$ and $\delta \bar{\sigma}$, and we have used formula (10) for $\delta \mathbf{n}$. The variations $\delta P \mathbf{n}-\delta \bar{\sigma} \cdot \mathbf{n}, \delta T$, and $\delta\left(\partial_{n} \hat{v}\right)$ appearing in Eq. (12) can be computed from the following linearized system:

$$
\begin{gathered}
\begin{cases}\delta R_{U}(U, \hat{v})=\frac{\partial R_{U}}{\partial U} \delta U+\frac{\partial R_{U}}{\partial \hat{v}} \delta \hat{v}=0 & \text { in } \Omega \\
\delta \mathbf{v}=-\partial_{n} \mathbf{v} \delta S & \text { on } S \\
\partial_{n}(\delta T)=(\nabla T) \cdot \nabla_{S}(\delta S)-\partial_{n}^{2} T \delta S & \text { on } S \\
(\delta W)_{+}=0 & \text { on } \Gamma_{\infty}\end{cases} \\
\begin{cases}\delta R_{\hat{v}}\left(U, \hat{v}, d_{S}\right)=\frac{\partial R_{\hat{v}}}{\partial U} \delta U+\frac{\partial R_{\hat{v}}}{\partial \hat{v}} \delta \tilde{v}+\frac{\partial R_{\hat{v}}}{\partial d_{S}} \delta d_{S}=0 & \text { in } \Omega \\
\delta \hat{v}=-\partial_{n} \hat{v} \delta S & \text { on } S \\
\delta \hat{v}=\sigma_{\infty} \delta v & \text { on } \Gamma_{\infty}\end{cases}
\end{gathered}
$$

$$
\begin{cases}\delta R_{d}\left(d_{S}\right)=\nabla d_{S} \cdot \nabla \delta d_{S}=0 & \text { in } \Omega \\ \delta d_{S}=\delta S & \text { on } S\end{cases}
$$

where $(\delta W)_{+}$represents the incoming characteristics on the far-field boundary. Linearization of the Navier-Stokes and the SpalartAllmaras equations are, respectively, given in Appendices $\mathrm{C}$ and $\mathrm{D}$. In the last formula of Eq. (16), we have used the fact that $\partial_{n} \bar{d}_{S}=-\overline{1}$; therefore, $\delta d_{S}=-\partial_{n} d_{S} \delta \overline{S=} \delta S$.

Domain integrals in Eq. (11) are eliminated using integration by parts and introducing the associated adjoint operators. This integration by parts also provides some boundary terms, which are combined with the boundary terms in Eq. (11) depending on $\delta P \mathbf{n}-\delta \sigma \cdot \mathbf{n}, \delta T$, and $\delta\left(\partial_{n} \hat{v}\right)$, yielding the boundary conditions for the adjoint operators. We describe this process below.

From Eqs. (14-16), the last three terms in Eq. (11) read

$$
\begin{aligned}
& \int_{\Omega} \Psi_{U}^{\top}\left(\frac{\partial R_{U}}{\partial U} \delta U+\frac{\partial R_{U}}{\partial \hat{v}} \delta \hat{v}\right)+\int_{\Omega} \psi_{\hat{v}}\left(\frac{\partial R_{\tilde{v}}}{\partial U} \delta U+\frac{\partial R_{\hat{v}}}{\partial \hat{v}} \delta \hat{v}+\frac{\partial R_{\hat{v}}}{\partial d_{S}} \delta d_{S}\right) \\
& +\int_{\Omega} \psi_{d} \frac{\partial R_{d}}{\partial d_{S}} \delta d_{S}=\int_{\Omega}\left(A_{U}^{U} \Psi_{U}+A_{\hat{v}}^{U} \psi_{\hat{v}}\right)^{\top} \delta U+\int_{\Omega}\left(A_{U}^{\hat{v}} \Psi_{U}+A_{\hat{v}}^{\hat{v}} \psi_{\hat{v}}\right) \delta \hat{v} \\
& +\int_{\Omega}\left(A_{\hat{v}}^{d} \psi_{\hat{v}}+A_{d}^{d} \psi_{d}\right) \delta d_{S}+\int_{S} B_{S} \mathrm{~d} s
\end{aligned}
$$

where $A_{U}^{U}=\left(\partial R_{U} / \partial U\right)^{\top}, \quad A_{\hat{v}}^{U}=\left(\partial R_{\hat{v}} / \partial U\right)^{\top}, \quad A_{U}^{\hat{v}}=\left(\partial R_{U} / \partial \hat{v}\right)^{\top}$, $A_{\hat{v}}^{\hat{v}}=\partial R_{\hat{v}} / \partial \hat{v}, A_{\hat{v}}^{d}=\partial R_{\hat{v}} / \partial d_{S}$, and $A_{d}^{d}=\partial R_{d} / \partial d_{S}$ are the adjoint operators and $B_{S}$ stands for the boundary terms coming from the integration by parts

$$
\begin{aligned}
& \int_{S} B_{S} \mathrm{~d} s=-\int_{S} \boldsymbol{\varphi} \cdot(\delta P \mathbf{n}-\delta \bar{\sigma} \cdot \mathbf{n}) \mathrm{d} s \\
& \quad-\int_{S}\left(\mathbf{g}_{1} \cdot \boldsymbol{\varphi}+g_{2} \partial_{n} \psi_{5}+\psi_{\hat{\nu}} g_{5}\right) \delta T \mathrm{~d} s-\int_{S} g_{3} \psi_{\hat{v}} \delta\left(\partial_{n} \hat{v}\right) \mathrm{d} s \\
& \quad+\int_{S} \psi_{\hat{\nu}} g_{4} \delta P \mathrm{~d} s-\int_{S} \psi_{d} \delta S \mathrm{~d} s+\int_{S} \hat{g} \delta S \mathrm{~d} s
\end{aligned}
$$

where $\boldsymbol{\varphi}=\left(\psi_{2}, \psi_{3}, \psi_{4}\right)$ and $\mathbf{g}_{1}, g_{i}, i=2,3,4,5$, are some functions that do not depend on the adjoint variables $\Psi_{U}, \psi_{\hat{v}}, \psi_{d}$, and $\hat{g}$, which does not depend on $\psi_{d}$. The analytical expression of these terms and the adjoint operators above are given in detail in Eqs. (E4-E13) in Appendix E.

We now observe that, due to the relation Eq. (6), the first and third terms on the right-hand side of Eq. (18) can be written together. In fact, the linearization of Eq. (6) provides

$$
\begin{aligned}
\delta P & =\delta(P \mathbf{n} \cdot \mathbf{n}-\mathbf{n} \cdot \bar{\sigma} \cdot \mathbf{n})=\mathbf{n} \cdot(\delta P \mathbf{n}-\delta \bar{\sigma} \cdot \mathbf{n}) \\
& -2(P \mathbf{n}-\mathbf{n} \cdot \bar{\sigma}) \cdot \nabla_{S}(\delta S) \quad \text { on } S
\end{aligned}
$$

where we have used the symmetry of the tensor $\bar{\sigma}$ and the formula for $\delta \mathbf{n}$ in Eq. (10). Therefore, Eq. (18) can be written as

$$
\begin{aligned}
\int_{S} & B_{S} \mathrm{~d} s=\int_{S}\left(-\boldsymbol{\varphi}+\psi_{\hat{\nu}} g_{4} \mathbf{n}\right) \cdot(\delta P \mathbf{n}-\delta \bar{\sigma} \cdot \mathbf{n}) \mathrm{d} s \\
- & \int_{S}\left(\mathbf{g}_{1} \cdot \boldsymbol{\varphi}+g_{2} \partial_{n} \psi_{5}+\psi_{\hat{\nu}} g_{5}\right) \delta T \mathrm{~d} s \\
- & \int_{S} g_{3} \psi_{\hat{\nu}} \delta\left(\partial_{n} \hat{v}\right) \mathrm{d} s-\int_{S} \psi_{d} \delta S \mathrm{~d} s-\int_{S} \hat{g} \delta S \mathrm{~d} s \\
& -2 \int_{S} \psi_{\hat{\nu}} g_{4}(P \mathbf{n}-\mathbf{n} \cdot \bar{\sigma}) \cdot \nabla_{S}(\delta S) \mathrm{d} s
\end{aligned}
$$

To eliminate domain integrals in Eq. (11) when replacing the last three terms by using Eq. (17), we assume that the adjoint variables satisfy

$$
\begin{aligned}
& 0=A_{U}^{U} \Psi_{U}+A_{\hat{v}}^{U} \psi_{\hat{v}} \\
& 0=A_{U}^{\hat{v}} \Psi_{U}+A_{\hat{v}}^{\hat{v}} \psi_{\hat{v}} \\
& 0=A_{\hat{v}}^{d} \psi_{\hat{v}}+A_{d}^{d} \psi_{d}
\end{aligned}
$$

Analogously, all boundary terms in Eq. (17) without explicit dependence on $\delta S$ can be eliminated by considering the following choice of boundary conditions for the adjoint variables:

$$
\begin{gathered}
\psi_{\hat{v}}=\frac{1}{g_{3}} \frac{\partial j}{\partial\left(\partial_{n} \hat{v}\right)} \text { on } S \\
\varphi_{i}=\frac{\partial j}{\partial f_{i}}+\psi_{\hat{v}} g_{4} n_{i} \text { on } S
\end{gathered}
$$

$$
\partial_{n} \psi_{5}=\frac{1}{g_{2}}\left(\frac{\partial j}{\partial T}+\mathbf{g}_{1} \cdot \boldsymbol{\varphi}+\psi_{\hat{\nu}} g_{5}\right) \quad \text { on } S
$$

$$
\psi_{d}=0 \quad \text { on } S
$$

Note that this choice for the boundary conditions must be done in an ordered manner; that is, the value of $\psi_{\hat{v}}$ on the right-hand sides of Eqs. (25) and (26) is obtained from Eq. (24), whereas the value of $\varphi$ in Eq. $(\overline{26})$ is computed from Eq. (25).

Combining Eqs. (11-13) and (17-20), adjoint equations (21-23), and the boundary conditions in Eqs. (24-27), we finally obtain

$$
\begin{aligned}
\delta \mathcal{J} & =\int_{S}\left(\frac{\partial j}{\partial f_{i}} \partial_{n} f_{i}+\frac{\partial j}{\partial T} \partial_{n} T+\frac{\partial j}{\partial\left(\partial_{n} \hat{v}\right)} \partial_{n}^{2} \hat{v}\right) \delta S \mathrm{~d} s \\
& -\int_{S}\left(\frac{\partial j}{\partial \mathbf{n}}+\frac{\partial j}{\partial \mathbf{f}} P-\frac{\partial j}{\partial \mathbf{f}} \cdot \bar{\sigma}\right) \cdot \nabla_{S}(\delta S) \mathrm{d} s \\
& -\int_{S}\left(\hat{g}+2 H_{m} j\right) \delta S \mathrm{~d} s-2 \int_{S} \psi_{\hat{v}} g_{4}(P \mathbf{n}-\mathbf{n} \cdot \bar{\sigma}) \cdot \nabla_{S}(\delta S) \mathrm{d} s
\end{aligned}
$$

In this expression, the adjoint variables are obtained by solving the closed system of PDEs and boundary conditions given by Eqs. (21-27).

It is important to recall here that neither the Navier-Stokes adjoint equation in Eq. (21) nor the Spalart-Allmaras adjoint equation in Eq. (22) depends on the adjoint distance variable $\psi_{d}$. This is also the case of the functional sensibility equation (28), since typical objective functionals in aerodynamics do not depend explicitly on the distance to the surface. In this situation, it is therefore not necessary to solve Eq. (23), and the adjoint system simply reduces to Eqs. (21) and (22). One could consider, however, more sophisticated situations that would require the solution of Eq. (23). This could be, 
for instance, the case of functionals with an explicit dependence on the distance to the surface by means of a domain integral.

Some particular but still interesting situations provide a more simplified formula for the variation of $J$, as described in [5] for the Navier-Stokes equations. Assume that the objective function depends only on $\mathbf{f}$ in the following way:

$$
j(\mathbf{f})=\mathbf{f} \cdot \mathbf{d}
$$

where $\mathbf{d}$ is a constant vector (the choice $\mathbf{d}=\mathbf{n}$ is also possible with some modifications, but for simplicity, we focus on constant $\mathbf{d}$ ). Note that this is the case in drag or lift optimization problems. The adjoint boundary conditions in this situation simply become

$$
\psi_{\hat{v}}=\psi_{d}=0, \quad \boldsymbol{\varphi}=\mathbf{d}, \quad \partial_{n} \psi_{5}=\mathbf{g}_{1} \cdot \mathbf{d} / g_{2}
$$

and the variation of $J$ is given by

$$
\begin{aligned}
\delta \mathcal{J} & =\int_{S} \mathbf{d} \cdot \partial_{n} f_{i} \delta S \mathrm{~d} s-\int_{S}(P \mathbf{d}-\mathbf{d} \cdot \bar{\sigma}) \cdot \nabla_{S}(\delta S) \mathrm{d} s \\
& -\int_{S}\left(\hat{g}+2 H_{m} j\right) \delta S \mathrm{~d} s
\end{aligned}
$$

Integrating now by parts, and assuming that either $S$ is smooth or $\delta S=0$ at its singular points, yields

$$
\begin{aligned}
\delta \mathcal{J} & =\int_{S} \partial_{n}(P \mathbf{d} \cdot \mathbf{n}-\mathbf{d} \cdot \bar{\sigma} \cdot \mathbf{n}) \delta S \mathrm{~d} s+\int_{S} \nabla_{S} \cdot(P \mathbf{d}-\mathbf{d} \cdot \bar{\sigma}) \delta S \mathrm{~d} s \\
& -\int_{S}\left(\hat{g}+2 H_{m} j\right) \delta S \mathrm{~d} s=\int_{S} \nabla \cdot(P \mathbf{d}-\mathbf{d} \cdot \bar{\sigma}) \delta S \mathrm{~d} s \\
& -\int_{S} \hat{g} \delta S \mathrm{~d} s=-\int_{S} \hat{g} \delta S \mathrm{~d} s
\end{aligned}
$$

Here, we have used the fact that the divergence operator, on local coordinates of $S$, is given by

$$
\nabla \cdot \mathbf{q}=\partial_{n}(\mathbf{q} \cdot \mathbf{n})+\nabla_{S} \cdot \mathbf{q}-2 H_{m} \mathbf{q} \cdot \mathbf{n}
$$

for a general vector field $\mathbf{q}$, and the identity

$$
\nabla \cdot(P \mathbf{d}-\mathbf{d} \cdot \bar{\sigma})=(\nabla P-\nabla \cdot \bar{\sigma}) \cdot \mathbf{d}=0 \quad \text { on } S
$$

which is obtained assuming that the momentum equations in the Navier-Stokes system are satisfied on the boundary, i.e., $\nabla P=\nabla \cdot \bar{\sigma}$ on $S$.

The final expression in Eq. (32) involves the function $\hat{g}$ that, as shown in Appendix E, is reduced to $\hat{g}=h$, given by Eq. (E12), due to the boundary conditions in Eq. (30). In this way, the expression for the total variation of the functional can be simplified as follows:

$$
\delta \mathcal{J}=-\int_{S} h \delta S \mathrm{~d} s=\int_{S}\left(\mathbf{n} \cdot \bar{\Sigma}^{\varphi} \cdot \partial_{n} \mathbf{v}-\mu_{\mathrm{tot}}^{2} C_{p} \nabla_{S} \psi_{5} \cdot \nabla_{S} T\right) \delta S \mathrm{~d} s
$$

with $\bar{\Sigma}^{\varphi}$ depending on the gradient of the adjoint variables $\varphi$. Note that we are supposing a smooth flow solution; a complete Euler adjoint formulation with shock waves can be found in [31].

\section{Numerical Implementation of the Shape Optimization Framework}

An appropriate rearrangement of terms in the vector-matrix multiplications in Eqs. (21) and (22) leads to a more suitable formulation for the numerical evaluation of the residuals of the adjoint system. Compact expressions for the evaluation of these terms, as well as a description of the other components of the whole shape optimization framework, are presented in this section.

\section{A. Spatial Discretization}

A finite volume discretization is used to solve both the direct and adjoint equations. As usual, the finite volume discretization is obtained by applying the integral formulation of the governing equations to a control volume $\Omega_{h}$, consisting of a cell of the dual mesh surrounding each node.

To avoid any possible confusion, the standard Latin indexes $i, j$ will be reserved here to indicate spatial coordinates. For those residuals computed as the addition of several fluxes through the faces of a dual mesh cell, the central node of the cell will be denoted with the local index 0 , and its set of neighboring points labeled as $\mathcal{N}_{0}=\left\{1, \ldots, N_{0}\right\}$, with $N_{0}$ representing the size of the set.

\section{Reynolds-Averaged Navier-Stokes Equations}

The solution of flow equation (1) and turbulence model equation (2) enter on the adjoint equations as the coefficients of the adjoint system. For the flow equations, a central scheme with a Jameson-Schmidt-Turkel (JST)-type scalar artificial dissipation $[32,33]$ is used for the discretization of the convective flux. The convection of the turbulent variable $\hat{v}$ is discretized using a fully upwinded scheme. Second-order accuracy is easily achieved via reconstruction of variables on the cell interfaces by using a MUSCL approach with limitation of gradients [34]. In both cases, viscous fluxes are computed with the node-gradient-based approach due to Weiss et al. [35] that, apart from reducing the truncation error of the scheme, avoids the odd-even decoupling of mesh nodes in the computation of residuals, resulting in second-order spatial accuracy. Source terms are approximated via piecewise reconstruction in the finite volume cells.

The solution of turbulence model equation (2) also requires the numerical approximation of the Eikonal Eq. (4), in order to compute the distance field to the boundary of the obstacle. Our implementation makes use of an efficient fast-marching solver for unstructured grids [36] based on a finite element approximation to the Eikonal equation in each mesh element. Second-order accuracy is recovered by using not only the information of the nodal values of the distance field but incorporating the direction of the computed $\nabla d_{S}$ into the solver [37].

\section{Adjoint Flow Equation}

a. Convective Residuals. A modified version of the JST scheme without low-order dissipation is used for the discretization of the convective term [5]. This is given by

$$
\mathcal{R}^{U, \text { conv }}=\int_{\Omega_{h}} \nabla \Psi_{U}^{\top} \cdot \mathbf{A}^{c} \mathrm{~d} \Omega \approx \sum_{n \in \mathcal{N}_{0}} \mathcal{R}_{0 n}^{U, \text { conv }}
$$

with $\mathbf{A}^{c}$ defined in Eq. (C6), which unfortunately is not written in conservative form, thus avoiding the direct application of the GreenGauss theorem. However, this flux can be seen as a convection with nonconstant coefficients, given by the evaluation of matrices $\mathbf{A}^{c}$ at the different mesh nodes. Hence, across the face of the control volume separating two mesh nodes with local indexes 0 and $n$, the component of the convective residual for node 0 can be computed as

$$
\mathcal{R}_{0 n}^{U, \text { conv }}=\left(\begin{array}{c}
(\mathbf{v} \cdot \mathbf{S}) \psi_{1}+\frac{|\mathbf{v}|^{2}}{2}(\gamma-1) l_{1 \psi}-(\mathbf{v} \cdot \mathbf{S}) l_{2 \psi} \\
(\mathbf{v} \cdot \mathbf{S}) \psi_{2}-v_{1}(\gamma-1) l_{1 \psi}+S_{1} l_{2 \psi} \\
(\mathbf{v} \cdot \mathbf{S}) \psi_{3}-v_{2}(\gamma-1) l_{1 \psi}+S_{2} l_{2 \psi} \\
(\mathbf{v} \cdot \mathbf{S}) \psi_{4}-v_{3}(\gamma-1) l_{1 \psi}+S_{3} l_{2 \psi} \\
(\mathbf{v} \cdot \mathbf{S}) \psi_{5}+(\gamma-1) l_{1 \psi}
\end{array}\right)+\mathcal{D}_{0 n}
$$

where the adjoint variables are reconstructed at the cell face as $\Psi_{U}=\frac{1}{2}\left(\left.\Psi_{U}\right|_{0}+\left.\Psi_{U}\right|_{n}\right)$, whereas flow variables are evaluated at node 0 (equivalently for node $n$ ). In Eq. (37), $\mathbf{S}=S_{i}=\left(S_{x}, S_{y}, S_{z}\right)$ denotes the normal vector of the face located at the edge going from node 0 to node $n$ such that its length coincides with the face area, and we have introduced the following notation:

$$
l_{1 \psi}=(\boldsymbol{\varphi} \cdot \mathbf{S})+(\mathbf{v} \cdot \mathbf{S}) \psi_{5}
$$




$$
l_{2 \psi}=\psi_{1}+(\varphi \cdot \mathbf{v})+H \psi_{5}
$$

The artificial dissipation between two nodes, 0 and 1 for example, can be expressed as

$$
\begin{gathered}
\mathcal{D}_{01}=\hat{\kappa}^{(4)} \varepsilon^{(4)}\left(\left.\nabla^{2} \Psi_{U}\right|_{0}-\left.\nabla^{2} \Psi_{U}\right|_{1}\right) \omega_{01} \lambda_{01} \\
\varepsilon^{(4)}=\left(3 \frac{N_{0}+N_{1}}{N_{0} N_{1}}\right)^{2} \\
\lambda_{0}=\left|\mathbf{v}_{0} \cdot \mathbf{S}\right|+c_{0}|\mathbf{S}| \\
\lambda_{01}=\left|\mathbf{v}_{01} \cdot \mathbf{S}\right|+c_{01}|\mathbf{S}| \\
\omega_{0}=\left(\frac{\lambda_{0}}{4 \lambda_{01}}\right)^{\frac{1}{2}} \\
\omega_{01}=\frac{\omega_{0} \omega_{1}}{\omega_{0}+\omega_{1}}
\end{gathered}
$$

where where the following abbreviations have been used:

$$
\Sigma_{i j}=\Sigma_{i j}^{\varphi}+\Sigma_{i j}^{5}
$$

$$
\Sigma_{i j}^{\varphi}=\mu_{\mathrm{tot}}^{1}\left(\partial_{j} \varphi_{i}+\partial_{i} \varphi_{j}-\frac{2}{3} \delta_{i j} \nabla \cdot \varphi\right)
$$

$$
\Sigma_{i j}^{5}=\mu_{\mathrm{tot}}^{1}\left(v_{j} \partial_{i} \psi_{5}+v_{i} \partial_{j} \psi_{5}-\frac{2}{3} \delta_{i j} \mathbf{v} \cdot \nabla \psi_{5}\right)
$$

$$
\begin{gathered}
\Sigma_{i}^{5}=\gamma \mu_{\mathrm{tot}}^{2} \partial_{i} \psi_{5} \\
\Sigma^{5}=\gamma \mu_{\mathrm{tot}}^{2} \nabla \psi_{5} \cdot \mathbf{S}
\end{gathered}
$$

For coherence with the discretization of the convective residuals, the gradients of the adjoint variables are averaged (making use of the Weiss correction) at the cell face, whereas flow variables are evaluated at node 0 .

c. Source Terms Residuals. The remaining terms in Eq. (E4) are treated as source terms and approximated via piecewise reconstruction of the solution in each finite volume cell. After some manipulations, the residual vector can be written as

$$
\mathcal{R}^{U, \text { source }} \approx\left|\Omega_{h}\right|\left(\begin{array}{c}
-\frac{1}{\rho} v_{i} \sigma_{i j} \partial_{j} \psi_{5}-\Sigma_{i j} \partial_{i}\left(\frac{v_{j}}{\rho}\right)+\left(\frac{|\mathbf{v}|^{2}}{2} \alpha_{j}-\beta_{j}\right) \partial_{j} \psi_{5}+\frac{1}{\rho} \Sigma_{j}^{5} v_{i} \partial_{j} v_{i}+\theta\left(|\mathbf{v}|^{2}-E\right)+\xi \\
\frac{1}{\rho} \sigma_{1 j} \partial_{j} \psi_{5}+\Sigma_{1 j} \partial_{j}\left(\frac{1}{\rho}\right)-v_{1} \alpha_{j} \partial_{j} \psi_{5}-\frac{1}{\rho} \Sigma_{j}^{5} \partial_{j} v_{1}-\theta v_{1} \\
\frac{1}{\rho} \sigma_{2 j} \partial_{j} \psi_{5}+\Sigma_{2 j} \partial_{j}\left(\frac{1}{\rho}\right)-v_{2} \alpha_{j} \partial_{j} \psi_{5}-\frac{1}{\rho} \Sigma_{j}^{5} \partial_{j} v_{2}-\theta v_{2} \\
\frac{1}{\rho} \sigma_{3 j} \partial_{j} \psi_{5}+\Sigma_{3 j} \partial_{j}\left(\frac{1}{\rho}\right)-v_{3} \alpha_{j} \partial_{j} \psi_{5}-\frac{1}{\rho} \Sigma_{j}^{5} \partial_{j} v_{3}-\theta v_{3} \\
\alpha_{j} \partial_{j} \psi_{5}+\theta
\end{array}\right)
$$

$$
\left.\nabla^{2} \Psi_{U}\right|_{0}=\sum_{n \mathcal{N}_{0}}\left(\left.\Psi_{U}\right|_{n}-\left.\Psi_{U}\right|_{0}\right)
$$

denotes the undivided Laplacian operator, $N_{1}$ represents the size of the set of neighboring points to node $1, \mathbf{v}_{01}=\frac{1}{2}\left(\left.\mathbf{v}\right|_{0}+\left.\mathbf{v}\right|_{1}\right)$ and $c_{01}=\frac{1}{2}\left(\left.c\right|_{0}+\left.c\right|_{1}\right)$ are the fluid and sound speeds at the cell face, and $\hat{\kappa}^{(4)}$ is an adjustable parameter. An artificial dissipation of upwind type could have been used as well, for which we refer the reader to [5].

b. Viscous Residuals. The viscous residuals of the adjoint flow equation are discretized using the Green-Gauss integral relation:

$$
\begin{aligned}
\mathcal{R}^{U, \text { visc }} & =\int_{\Omega_{h}} \nabla\left(\nabla \Psi_{U}^{\top} \cdot \mu_{\mathrm{tot}}^{k} \mathbf{D}^{v k}\right) \mathrm{d} \Omega \\
& =\int_{\partial \Omega_{h}} \nabla \Psi_{U}^{\top} \cdot \mu_{\mathrm{tot}}^{k} \mathbf{D}^{v k} \mathrm{~d} \mathbf{S} \approx \sum_{n \in \mathcal{N}_{0}} \mathcal{R}_{0 n}^{U, \text { visc }}
\end{aligned}
$$

with $\mathbf{D}^{v k}$ defined in Eq. (C6), which yields the following form for the viscous flux:

$$
\mathcal{R}_{0 n}^{U, \text { visc }}=\frac{1}{\rho}\left(\begin{array}{c}
-v_{i} \Sigma_{i j} S_{j}+\left(\frac{|\mathbf{v}|^{2}}{2}-\frac{P}{(\gamma-1) \rho}\right) \Sigma^{5} \\
\Sigma_{1 j} S_{j}-v_{1} \Sigma^{5} \\
\Sigma_{2 j} S_{j}-v_{2} \Sigma^{5} \\
\Sigma_{3 j} S_{j}-v_{3} \Sigma^{5} \\
\Sigma^{5}
\end{array}\right)
$$

where $\left|\Omega_{h}\right|$ denotes the cell measure, and

$$
\begin{gathered}
\alpha_{i}=\gamma \mu_{\mathrm{tot}}^{2} \partial_{i}\left(\frac{1}{\rho}\right) \\
\beta_{i}=\frac{\gamma}{\gamma-1} \mu_{\mathrm{tot}}^{2} \partial_{i}\left(\frac{P}{\rho^{2}}\right) \\
\kappa_{\psi}=\bar{\tau}: \nabla \boldsymbol{\varphi}+\mathbf{v} \cdot \bar{\tau} \cdot \nabla \psi_{5}+\frac{C_{p}}{P r_{t}} \nabla T \cdot \nabla \psi_{5} \\
\theta=\left[\kappa_{\psi}\left(1-\frac{\mu_{\mathrm{tur}}}{\mu_{\mathrm{dyn}}} \frac{3 c_{v 1}^{3}}{\chi^{3}+c_{v 1}^{3}}\right)\right. \\
\left.-\frac{C_{p}}{P r_{t}} \nabla T \cdot \nabla \psi_{5}\left(1-\frac{P r_{t}}{P r_{d}}\right)\right] \frac{(\gamma-1)}{R \rho} \frac{\partial \mu_{\mathrm{dyn}}}{\partial T} \\
\xi=\kappa_{\psi}\left(1+\frac{3 c_{v 1}^{3}}{\chi^{3}+c_{v 1}^{3}}\right) \frac{\mu_{\mathrm{tur}}}{\rho}
\end{gathered}
$$

and $\bar{\tau}: \nabla \varphi=\tau_{i j} \partial_{i} \varphi_{j}$, with $\partial \mu_{\text {dyn }} / \partial T$ given by Eq. (C3).

d. Coupling Residuals. Coupling between the Navier-Stokes and the Spalart-Allmaras adjoint equations is obtained by means of Eq. (E5). Its two first terms are integrated using piecewise (p.w.) reconstruction, yielding a combined residual 


$$
\mathcal{R}^{U \text {,coupling }\left.\right|_{\text {p.w. }} \approx\left|\Omega_{h}\right|}\left(\begin{array}{c}
-\frac{\Gamma}{\rho}\left(\tilde{\alpha}\left(|\mathbf{v}|^{2}-E\right)-\frac{\mu_{\mathrm{dyn}}}{\rho}\right)+\frac{\hat{v}}{\rho} v_{j} \partial_{j} \psi_{\hat{v}}+\psi_{\hat{v}} \frac{\Lambda}{|\omega|}\left(\partial_{j} v_{i}-\partial_{i} v_{j}\right) \partial_{j}\left(\frac{v_{i}}{\rho}\right) \\
\frac{\Gamma}{\rho} \tilde{\alpha} v_{1}-\frac{\hat{v}}{\rho} \partial_{1} \psi_{\hat{v}}-\psi_{\hat{v}} \frac{\Lambda}{|\omega|}\left(\partial_{j} v_{1}-\partial_{1} v_{j}\right) \partial_{j}\left(\frac{1}{\rho}\right) \\
\frac{\Gamma}{\rho} \tilde{\alpha} v_{2}-\frac{\hat{v}}{\rho} \partial_{2} \psi_{\hat{v}}-\psi_{\hat{v}} \frac{\Lambda}{|\omega|}\left(\partial_{j} v_{2}-\partial_{2} v_{j}\right) \partial_{j}\left(\frac{1}{\rho}\right) \\
\frac{\Gamma}{\rho} \tilde{\alpha} v_{3}-\frac{\hat{v}}{\rho} \partial_{3} \psi_{\hat{v}}-\psi_{\hat{v}} \frac{\Lambda}{|\omega|}\left(\partial_{j} v_{3}-\partial_{3} v_{j}\right) \partial_{j}\left(\frac{1}{\rho}\right) \\
-\frac{\Gamma}{\rho} \tilde{\alpha}
\end{array}\right)
$$

with the abbreviations

$$
\begin{gathered}
\tilde{\alpha}=\frac{(\gamma-1)}{R \rho} \frac{\partial \mu_{\mathrm{dyn}}}{\partial T} \\
\Gamma=\psi_{\hat{v}} \Lambda \hat{S}^{f_{v 2}}\left(f_{v 2}^{\chi}+f_{v 2}^{v 1} f_{v 1}^{\chi}\right) \chi^{v}-\frac{1}{\sigma} \nabla \hat{v} \cdot \nabla \psi_{\hat{v}}
\end{gathered}
$$

and all partial derivatives appearing in these formulas are given in Appendix D.

The third term of Eq. (E5) is, however, written in conservative form. Hence, this part of the residual is evaluated by means of the Green-Gauss theorem as

$$
\left.\mathcal{R}^{U, \text { coupling }}\right|_{\text {cons }} \approx \sum_{n \in \mathcal{N}_{0}} \frac{\psi_{\hat{v}}}{\rho} \frac{\Lambda}{|\boldsymbol{\omega}|}\left(\begin{array}{c}
-v_{i}\left(\partial_{j} v_{i}-\partial_{i} v_{j}\right) S_{j} \\
\left(\partial_{j} v_{1}-\partial_{1} v_{j}\right) S_{j} \\
\left(\partial_{j} v_{2}-\partial_{2} v_{j}\right) S_{j} \\
\left(\partial_{j} v_{3}-\partial_{3} v_{j}\right) S_{j} \\
.
\end{array}\right)
$$

\section{Adjoint Turbulent Equation}

a. Convective Residuals. To preserve consistency with the direct solver, the turbulent adjoint variable $\psi_{\hat{v}}$ is discretized using a second-order upwind scheme with face reconstruction and limitation of gradients. This convection is also given as a nonconservative flux in the form

$$
\mathcal{R}^{\hat{v}, \text { conv }}=\int_{\Omega_{h}} \nabla \psi_{\hat{v}} \cdot \mathbf{B}^{c v} \mathrm{~d} \Omega \approx \sum_{n \in \mathcal{N}_{0}} \mathcal{R}_{0 n}^{\hat{v}, \text { conv }}
$$

where the analytical expression for the flux $\mathbf{B}^{c v}$ is given by Eq. (D4). Supposing a regular solution of the adjoint equations, the convective flux for node 0 across the face of the control volume separating nodes 0 and $n$ is then approximated as

$$
\mathcal{R}_{0 n}^{\hat{v}, \text { conv }}=\frac{1}{2}\left[\mathbf{B}_{0}^{c v} \cdot \mathbf{S}\left(\left.\psi_{\hat{v}}\right|_{0}+\left.\psi_{\hat{v}}\right|_{n}\right)-\left|\mathbf{B}_{0 n}^{c v} \cdot \mathbf{S}\right|\left(\left.\psi_{\hat{v}}\right|_{n}-\left.\psi_{\hat{v}}\right|_{0}\right)\right]
$$

b. Viscous Residuals. Viscous residuals are newly discretized using the Green-Gauss integral relation:

$$
\mathcal{R}^{\hat{v}, \text { visc }}=\int_{\Omega_{h}} \nabla\left(\nabla \psi_{\hat{v}} \cdot \mathbf{E}^{c v}\right) \mathrm{d} \Omega=\int_{\partial \Omega_{h}} \nabla \psi_{\hat{v}} \cdot \mathbf{E}^{c v} \mathrm{~d} \mathbf{S} \approx \sum_{n \in \mathcal{N}_{0}} \mathcal{R}_{0 n}^{\hat{v}, \text { visc }}
$$

with $\mathbf{E}^{c v}$ defined in Eq. (D5), yielding for node 0 the following flux:

$$
\mathcal{R}_{0 n}^{\hat{v}, \text { visc }}=-\frac{v+\hat{v}}{\sigma} \nabla \psi_{\hat{v}} \cdot \mathbf{S}
$$

where all the variables and the gradients of the turbulent adjoint variable, including the Weiss correction, are averaged at the cell face.

c. Source Terms Residuals. The rest of the terms in Eq. (E7) constitute the source contributions to the adjoint turbulent equation. As for the coupling residuals of the adjoint Navier-Stokes equation, the two first terms are integrated using piecewise reconstruction, resulting in a residual in the form

$$
\left.\mathcal{R}^{\hat{v}, \text { source }}\right|_{\text {p.w. }} \approx\left|\Omega_{h}\right|\left(-\psi_{\hat{v}} B^{s}\right)
$$

with $B^{s}$ given by Eq. (ㄱ). The $\mathbf{E}^{s}$ term is evaluated by the GreenGauss integral relation, yielding

$$
\left.\mathcal{R}^{\hat{v}, \text { source }}\right|_{\text {cons }} \approx \sum_{n \in \mathcal{N}_{0}} \frac{2 c_{b 2}}{\sigma} \psi_{\hat{v}} \nabla \hat{v} \cdot \mathbf{S}
$$

d. Coupling Residuals. Finally, residuals coming from the coupling with the adjoint flow equation, given by Eq. (E6), can be written as

$$
\mathcal{R}^{\hat{v}, \text { coupling }} \approx\left|\Omega_{h}\right| \kappa_{\psi} \frac{\partial \mu_{\text {tur }}}{\partial \hat{v}}
$$

with $\kappa_{\psi}$ and $\partial \mu_{\text {tur }} / \partial \hat{v}$ given by Eqs. (드) and (드).

\section{Adjoint Eikonal Equation}

As previously stated, neither the adjoint flow nor the adjoint turbulent equations depend on the adjoint distance variable $\psi_{d}$. Furthermore, this adjoint variable is not needed either to compute the functional sensitivity, since typical objective functionals in aerodynamics do not depend explicitly on the distance to the surface. Hence, for the functionals considered here, there is no need to implement and numerically solve Eq. (23).

\section{Boundary Conditions}

Boundary conditions for a solid wall can be imposed in two ways: either by using a ghost cell scheme adapted to unstructured meshes or by directly enforcing the boundary conditions on the analytical flux expressions. On the far field, characteristic boundary conditions are used.

\section{B. Steady-State Time Integration}

A time-marching strategy in pseudotime $[33,38]$ is used to obtain the steady solution of the flow equations and the adjoint system equations (21) and (22). Although the two sets of equations are coupled over the turbulent viscosity $\mu_{\text {tur }}$, and it would be more efficient in terms of operation counts to formulate and solve them simultaneously, decoupling is by far the most widely used strategy, both for simplicity and because of the different character of both types of equations. In addition, it also gives flexibility in order to introduce other models of turbulence in a future.

Because of the inherent stiffness of turbulence-transport equations, time integration of both the Navier-Stokes and the Spalart-Allmaras adjoint systems is tackled with an implicit backward-Euler scheme [19]. Direct inversion of the banded matrix that defines this system is impractical because of rapid increase of operation counts with the number of mesh points and large storage requirements, especially in three dimensions. The resulting linear system is therefore solved iteratively by means of a lower-upper symmetric Gauss-Seidel algorithm [14,39]. To speed up the rate of convergence, an overset multigrid scheme has been implemented in conjunction with the solver $[40,41]$.

\section{Design Variables}

In the present work, the shape functions introduced by Hicks and Henne [42] have been used as design variables for the two- 


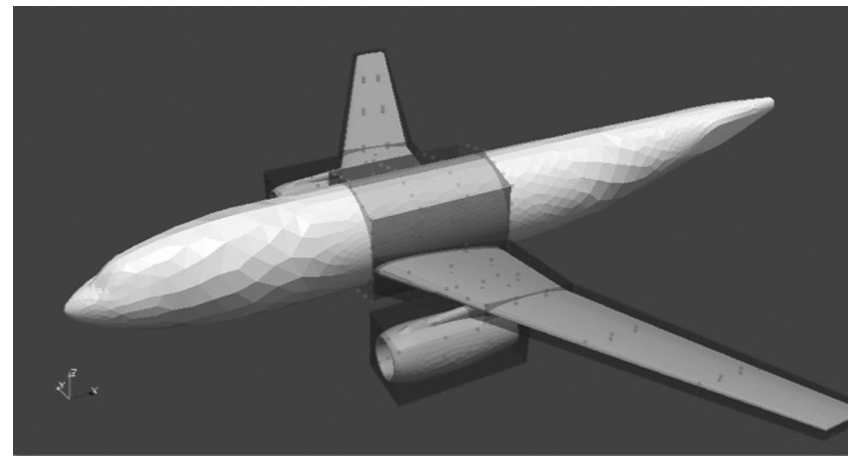

Fig. 1 Example of FFD boxes on a DLR-F6 aircraft configuration.

dimensional (2-D) examples. The Hicks-Henne function with maximum at point $x_{n}$ is given by

$$
f_{n}(x)=\sin ^{3}\left(\pi x^{e_{n}}\right), \quad e_{n}=\frac{\log (0.5)}{\log \left(x_{n}\right)}, \quad x \in[0,1]
$$

so the total deformation of the surface can be computed as

$$
\Delta y=\sum_{n=1}^{N} \delta_{n} f_{n}(x)
$$

with $N$ being the number of bump functions and $\delta_{n}$ the design variable step. These functions are applied separately to the upper and lower surfaces.

For three-dimensional (3-D) problems, a freeform deformation (FFD) strategy has been adopted [43]. In FFD, an initial box encapsulating the object (wing, airplane, etc.) that one wants to redesign is parameterized as a Bézier solid (Fig. 1). A set of control points is defined on the surface of the box, depending on the order of the chosen Bernstein polynomials. In this way, the solid box is parameterized by the following expression:

$$
X(u, v, w)=\sum_{i, j, k=0}^{l, m, n} P_{i, j, k} B_{i}^{l}(u) B_{j}^{m}(v) B_{k}^{n}(w)
$$

where $u, v, w \in[0,1]$, and $B^{i}$ is the Bernstein polynomial of order $i$. The Cartesian coordinates of the points on the surface of the object are transformed to the parametric coordinates of the Bézier box. Control points of the box become design variables, as they control the shape of the solid and thus the shape of the surface grid inside. The embedding box is deformed by modifying its control points, with all the points inside the box inheriting a smooth deformation. The new Cartesian coordinates of the object of interest can then be recovered by inverting mapping equation (71).

Finally, once the boundary displacements have been computed, a torsional spring method $[44,45]$ is used in order to reallocate the rest of vertexes of the unstructured mesh.

\section{Optimization Framework}

The optimization results presented in this work make use of the SciPy library,- a well-established open-source software for mathematics, science, and engineering. At each design iteration, the SciPy routines only require as inputs the values and gradients of the objective functions, computed by means of our continuous adjoint approach, as well as the set of chosen constraints.

\section{Summary of Results and Numerical Experiments}

The adjoint formulation presented in this paper has been implemented in the aerodynamic shape design suite CADES (code for aerodynamical design and simulation, developed by the research group). CADES is a complete suite for aerodynamical shape design composed by five $\mathrm{C}++$ programs [computational fluid dynamics (CFD) solver, grid adaptation, grid deformation, domain

\footnotetext{
IData available at http://www.scipy.org [retrieved 2011].
}

partitioning, and gradients computation] and several Python scripts. In particular, the software CADES_CFD is a finite volume code that solves the direct (flow), adjoint, and linearized problems for the potential, Euler, Navier-Stokes, and RANS equations on either 2-D or 3-D unstructured meshes using an edge-based data structure.

All terms of the turbulent adjoint formulation were implemented in the software suite with the exception of those involving the factor $\Lambda /|\omega|$ in Eqs. (59) and (62). Numerical inspection of $\Lambda$, given by Eq. (D12), reveals very small values for this quantity, with a fast decay outside the turbulent boundary layer. Precisely, the magnitude of the vorticity is greater close to the airfoil surface, making the whole factor $\Lambda /|\omega|$ negligible when compared with the rest of terms of the adjoint residuals. However, neglecting these terms helps to avoid numerical instabilities far from the obstacle surface, where the magnitude of the vorticity tends to zero. Other regularization approaches for these terms are under current development.

A weighted least-squares method was used in our calculations to approximate the spatial gradients of the flow and the adjoint variables, although similar results were obtained by using the GreenGauss formulation. Standard values for the first-, second-, and fourth-order artificial dissipation coefficients of the flow JST solver (parameters $\kappa^{(0)}, \kappa^{(2)}$, and $\kappa^{(4)}$ in $\left.[32,33]\right)$ are $0.1,1 / 4$, and $1 / 64$, respectively. The value for the adjoint fourth-order artificial dissipation coefficient $\hat{\kappa}^{(4)}$ in Eq. (40) is taken to be $1 / 150$. This value allows all our simulations to be numerically stable while introducing minimum artificial dissipation effects in the results. In the calibration procedure, we have used finite difference results and a grid convergence study with the adjoint Euler equations.

This section presents some numerical tests regarding the solution of the continuous turbulent adjoint problem. First, gradients computed with the new adjoint method are compared with those obtained using the so-called frozen viscosity hypothesis. This hypothesis assumes that variations of the fluid viscosity with respect to the flow variables can be neglected when compared with the rest of terms of the flow adjoint equation. Thus, the turbulence model is used to compute the flow variables, but only the adjoint equation for the mean flow is considered; that is, it solves

$$
0=A_{U}^{U} \Psi_{U}
$$

subject to

$$
\begin{gathered}
\varphi_{i}=\frac{\partial j}{\partial f_{i}} \quad \text { on } S \\
\partial_{n} \psi_{5}=\frac{1}{g_{2}}\left(\frac{\partial j}{\partial T}+\mathbf{g}_{1} \cdot \boldsymbol{\varphi}\right) \quad \text { on } S
\end{gathered}
$$

also neglecting the last term in Eq. (E4) for the computation of the adjoint operator $A_{U}^{U}$.

Then, a transonic unconstrained drag minimization problem is shown to highlight the importance of using the complete adjoint methodology compared with the frozen viscosity strategy. Finally, an unconstrained turbulent 3-D drag minimization problem using the FFD technique will be presented to study the viability of FFD for the definition of 3-D design variables.

\section{A. Numerical Comparison of Gradients in Drag and Lift Optimization Problems}

Our first case of study involves a transonic, turbulent flow over the RAE-2822 airfoil. The flow conditions correspond to the AGARDAR-138 case 9 [46], with corrections to account for wind-tunnel effects [47], namely, $M_{\infty}=0.734, \alpha=2.54^{\circ}$, and $R e=6.5 \times 10^{6}$. Under these conditions, the flow develops a shock wave on the upper surface (located at about 50 to $60 \%$ of the airfoil chord) and a very small shock-induced separation behind the shock. The computational grid is an unstructured 2-D grid with 13,937 nodes and 22,842 elements, with 192 nodes on the airfoil surface and 40 nodes on the far-field boundary, which is located 100 chords away from the airfoil.

The Hicks-Henne functions are used as design variables in this example. The first design variable has its maximum close to the 
trailing edge on the lower side of the airfoil, and subsequent variables displace the maximum in the clockwise direction. A total number of 38 bump functions were used, spanning the complete surface of the airfoil. Gradients computed with the two adjoint methods are compared with those obtained with a forward finite difference method. The design variable step $\delta_{n}$ was decreased until convergence of the finite difference gradients was achieved. In these computations, a value of $\delta_{n}=10^{-8}$ was used to generate the reference gradients.

Figure 2 summarizes our main results on the comparison of gradients for the drag $c_{d}$ and lift $c_{l}$ functionals. In this figure, the negative part of the $x$ axis denotes design variables located on the lower side of the airfoil, and positive $x$ denotes those located on the upper side. Assuming the finite differences approximation as the reference solution, it is clear that the gradients computed by the turbulent adjoint method described in this paper are in much better agreement with the exact solution than those obtained using the frozen viscosity simplification. Moreover, as can be seen for both functionals, on the lower surface of the airfoil close to the trailing edge, the frozen viscosity adjoint can indeed predict a gradient not only wrong in magnitude but also in its direction, thus seriously compromising the efficiency of a gradient-based optimization solver during the minimization process.

The left panels of Fig. $\underline{3}$ (drag) and Fig. 4 (lift) present the convergence histories of the density-adjoint variable $\psi_{1}$ (left axes/ solid lines) and the geometrical sensitivity parameter (right axes/ dashed lines) given by the term that multiplies $\delta S$ in gradient formula equation (35) for both the turbulent and the frozen viscosity adjoint methods. Convergence improvements are especially relevant for the computation of lift gradients, although no substantial reduction in the number of iterations is obtained in this case for drag calculations. The right panels of these figures show the computed fields of the density-adjoint variable for the two methods under study. Note that,
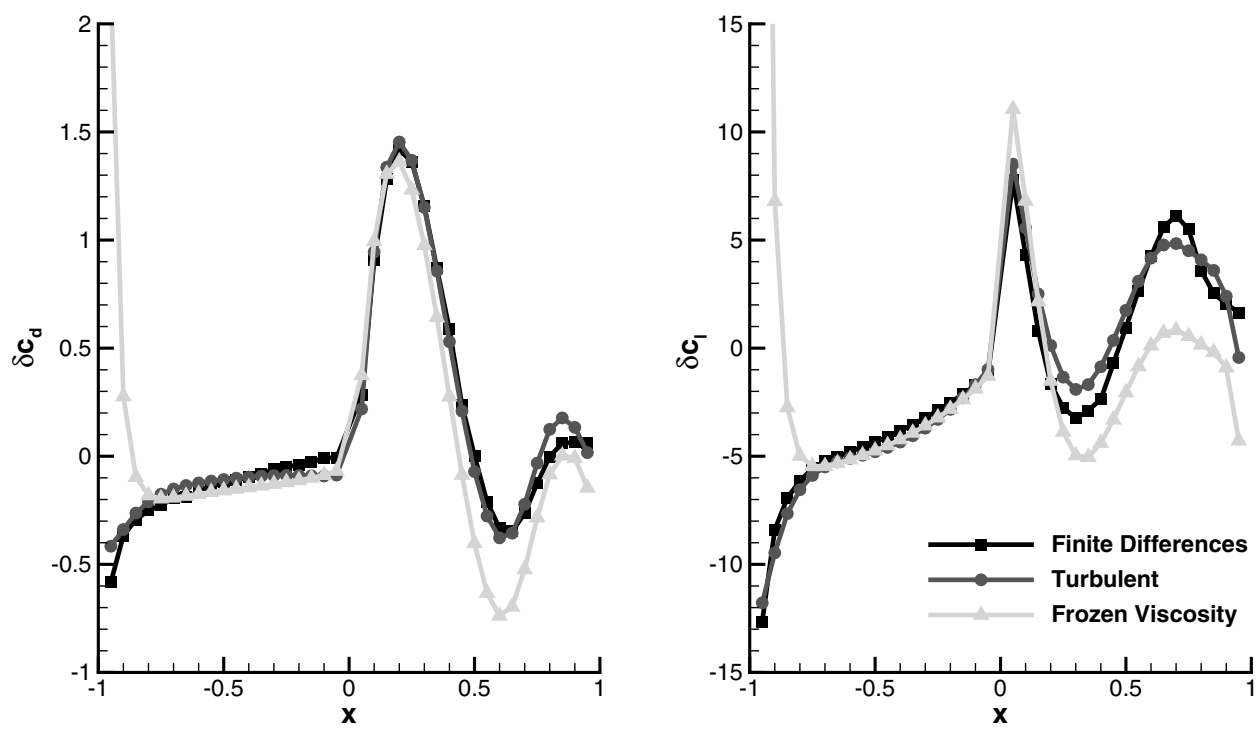

Fig. 2 Comparison of gradients for drag (left) and lift (right) coefficients for the RAE-2822 airfoil.

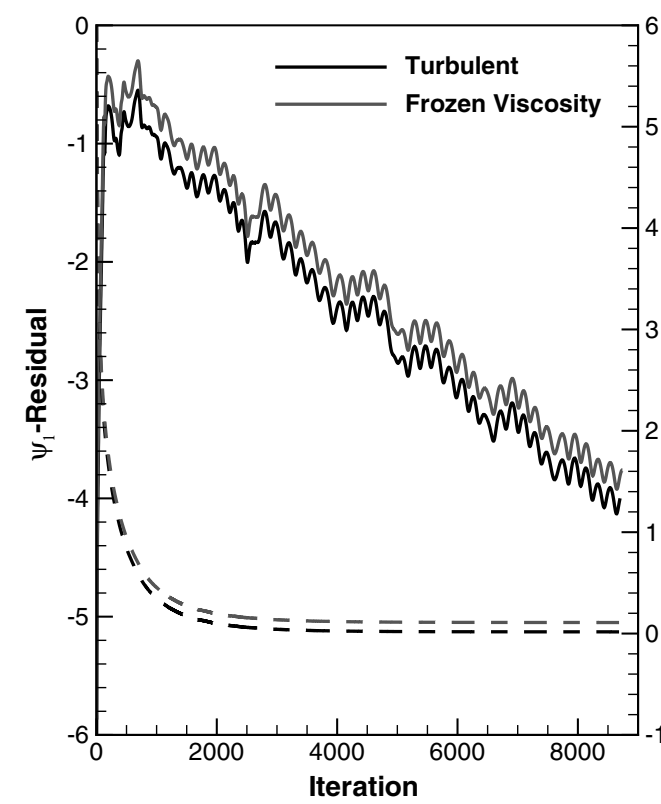

a) Convergence history of the turbulent and frozen viscosity adjoint methods
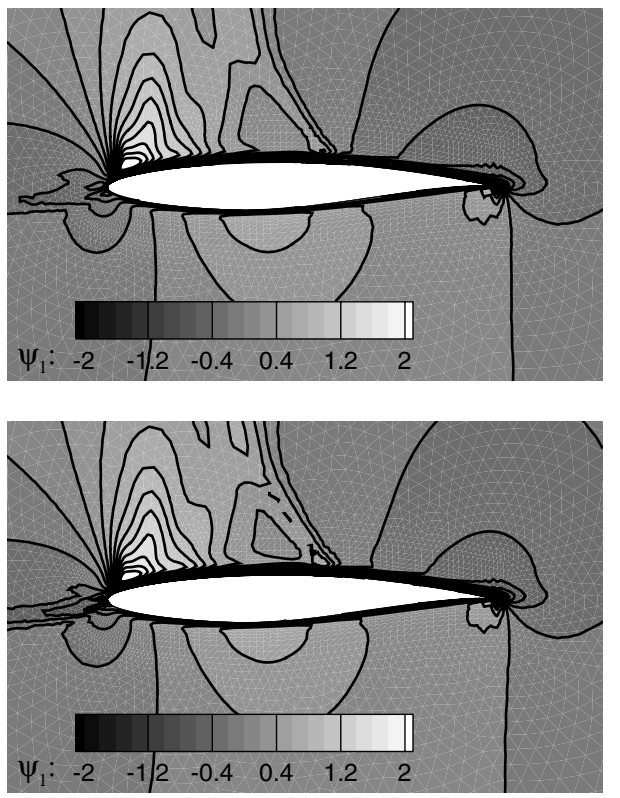

b) Density-adjoint variable fields for drag objective function using the turbulent (top) and the frozen viscosity (bottom) adjoint methods

Fig. 3 Comparison between the turbulent and the frozen viscosity adjoint methods in the computation of drag sensitivities. Traces for the frozen viscosity approach have been slightly shifted in Fig. 3a to avoid overlapping. 


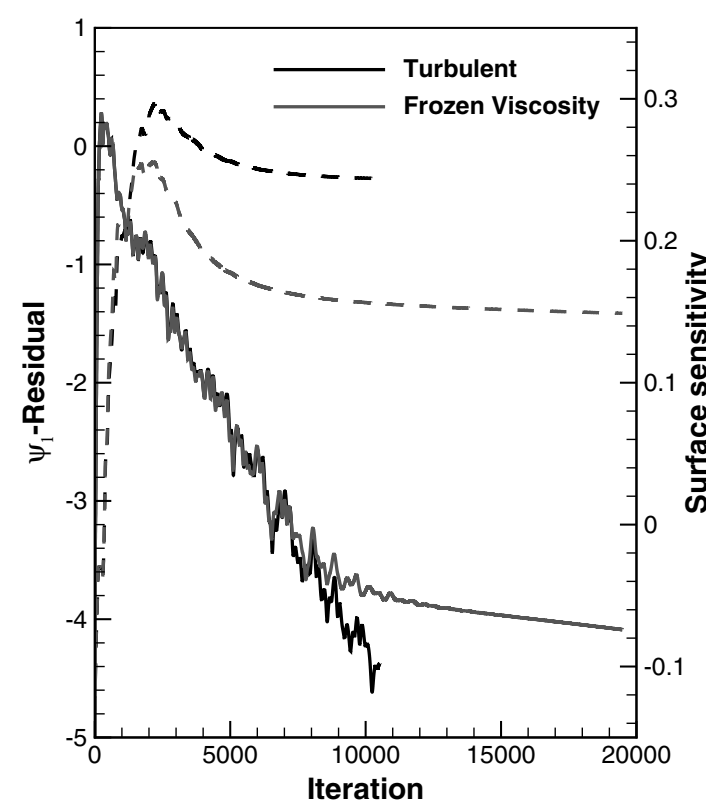

a) Convergence history of the turbulent and frozen viscosity adjoint methods
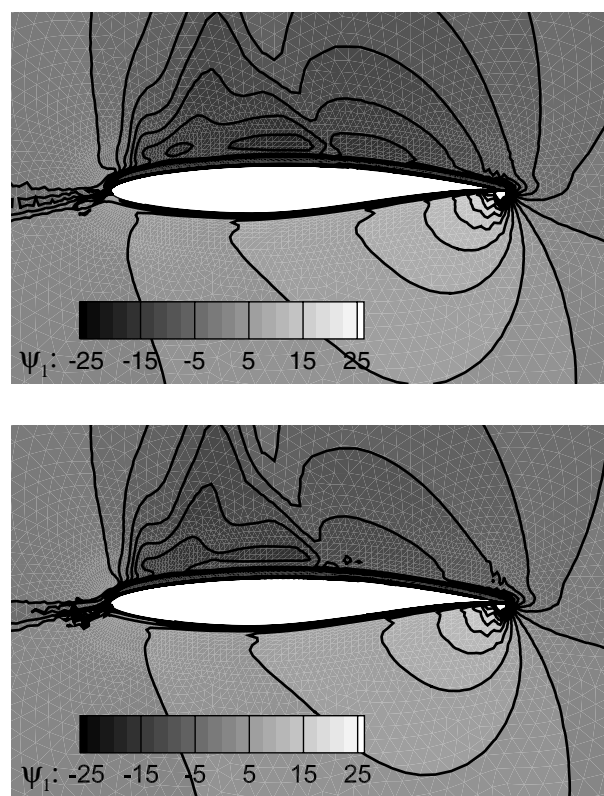

b) Density-adjoint variable fields for lift objective function using the turbulent (top) and the frozen viscosity (bottom) adjoint methods

Fig. 4 Comparison between the turbulent and the frozen viscosity adjoint methods in the computation of lift sensitivities.

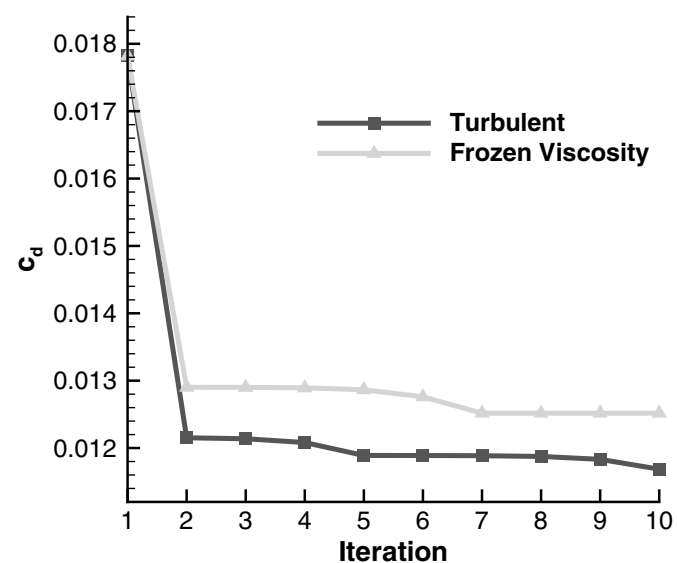

a) Optimization convergence history, turbulent adjoint method vs frozen viscosity

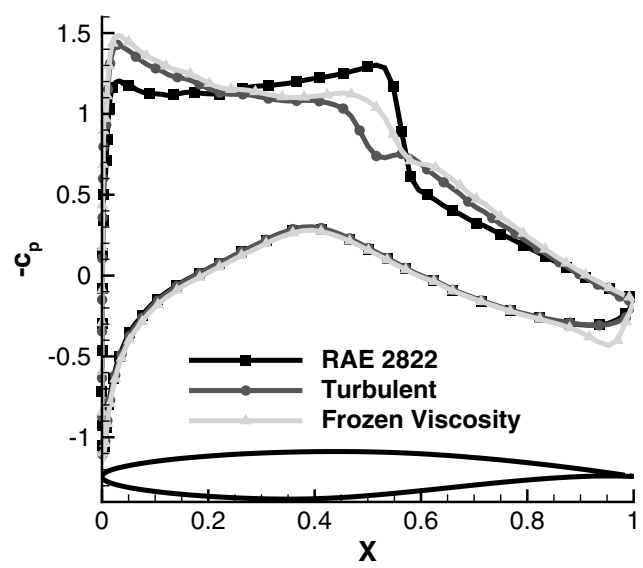

b) Pressure coefficient distribution of the initial and redesigned RAE-2822 airfoil

Fig. 5 Unconstraint drag minimization of a RAE-2822 airfoil (transonic flow modeled by the RANS equations).

in general, the solutions of the complete turbulent adjoint are somehow less affected by the presence of the shock wave. The same applies for the rest of adjoint flow variables.

\section{B. Two-Dimensional Unconstrained Drag Minimization Using Adjoint Reynolds-Averaged Navier-Stokes}

The goal of this academic problem is to reduce the drag of the RAE-2822 profile by means of modifications of its surface. The angle of attack, Mach number, and Reynolds number are kept fixed so that the flow remains transonic $\left(M_{\infty}=0.734, \alpha=2.54^{\circ}\right.$, and $\left.R e=6.5 \times 10^{6}\right)$. The same set of 38 Hicks-Henne functions defined in the previous section has been used here as design variables.

The convergence of the optimization process is presented in the left panel of Fig. 5. As expected, gradients computed with the complete adjoint Spalart-Allmaras formulation provide an airfoil with a lower value of drag coefficient than using the frozen viscosity gradients (note the nonoptimal pressure distribution of the trailing edge due to the erroneous frozen viscosity gradient at that location). The initial pressure distribution and the optimized one with the Spalart-Allmaras adjoint method are shown on the right panel of this

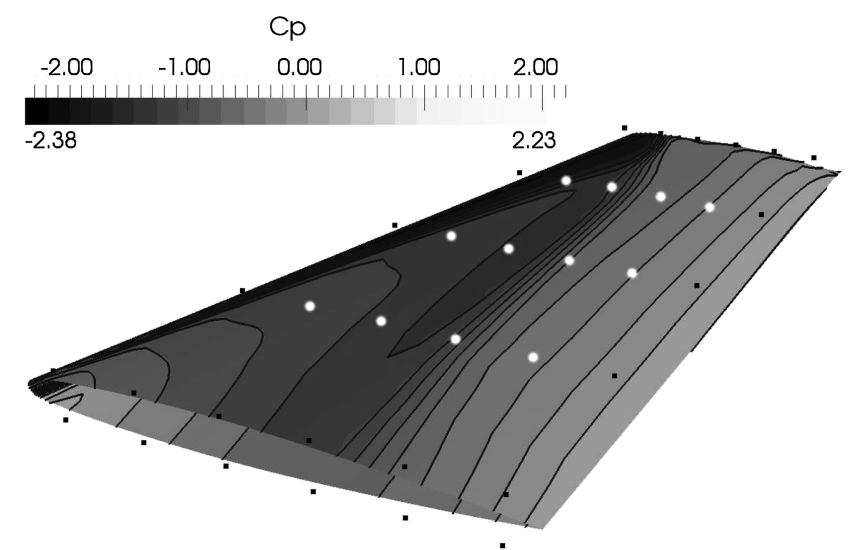

Fig. 6 Pressure coefficient distribution of the ONERA-M6 wing and control points of the FFD box (white points are design variables). 

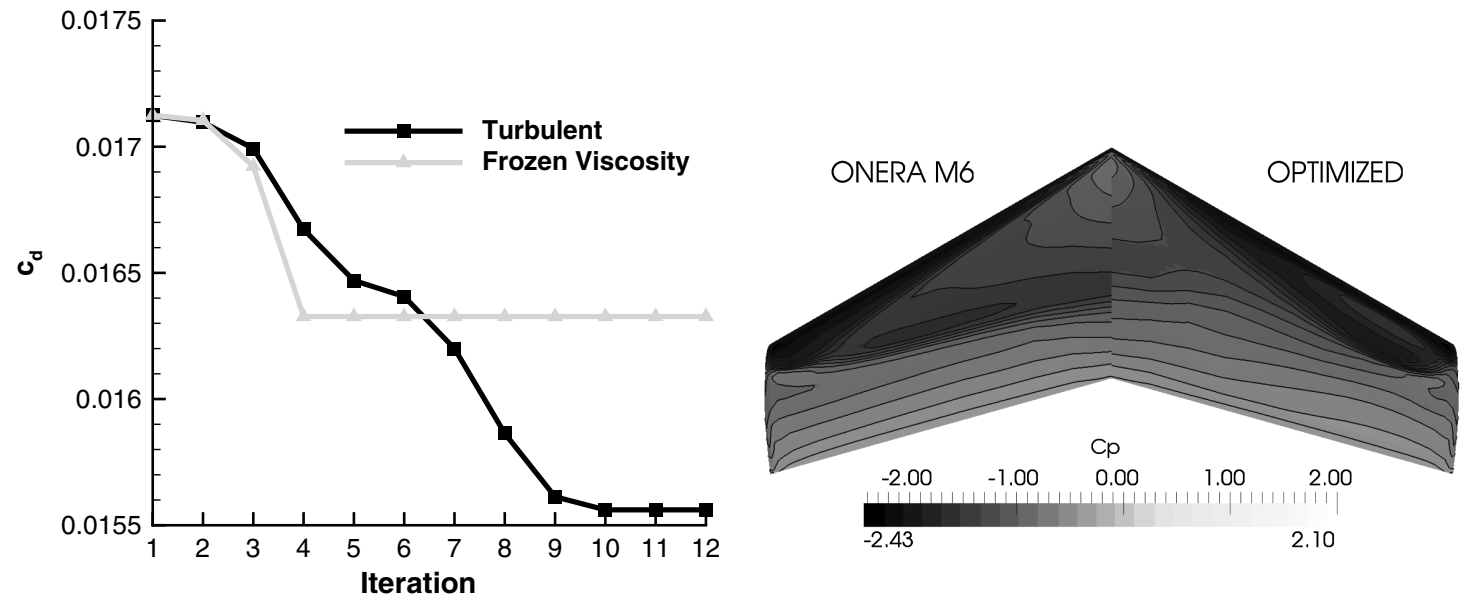

Fig. 7 Optimization convergence history, turbulent adjoint method vs frozen viscosity (left). Pressure coefficient distribution of the initial and redesigned ONERA-M6 airfoil (right).

figure. In particular, the original drag coefficient was 0.0178 , and the final one was 0.0116 , representing a total $34.8 \%$ drag reduction.

\section{Three-Dimensional Unconstrained Drag Minimization Using} Adjoint Reynolds-Averaged Navier-Stokes and Freeform Deformation

The objective of our last numerical test is to check the performance of the developed methodology in conjunction with the FFD approach for the definition of 3-D design variables. A single-point minimization case is used to study the new continuous adjoint method for turbulent flows. The selected flow conditions were $M_{\infty}=0.8395$, $\alpha=3.06^{\circ}$, and $R e=11.72 \times 10^{6}$, and the aerodynamic profile was an ONERA-M6 wing (43,008 hexahedron elements). Only the upper surface of the wing will be redesigned in this case. A total number of 12 control points are used as design variables (see white points in Fig. 6), employing Bernstein polynomials of the sixth, fifth, and second orders in the $x, y$, and $z$ directions, respectively.

To determine convergence, the drag and the sensitivity coefficients were monitored on the surface of the wing profile. A Cauchy's convergence criteria for series in both coefficients (no changes in their sixth decimal place during 100 iterations) was used as stopping criteria in this example. The entire design process (12 optimization iterations) was performed overnight using a personal laptop (2.53 GHz Intel Core $2 \mathrm{Duo}$ ).

Figure 7 (left) shows a comparison of the optimization convergence history between the frozen viscosity strategy and the complete adjoint formulation for our 3-D case of study. Note that the frozen viscosity strategy does not result into any design improvement after the fourth iteration of the optimization process, achieving only a 5\% drag reduction. On the other hand, after 10 iterations of the complete turbulent adjoint method, the drag coefficient is finally reduced a total of $10 \%$. The right panel of the same figure also shows the pressure coefficient distribution of the original ONERA-M6 wing compared with the redesigned profile using the complete SpalartAllmaras adjoint formulation. The shock wave initially present on its upper surface has been clearly diminished in the redesigned airfoil. Hence, this optimal design test proves the flexibility of the continuous adjoint approach in 3-D applications, combined with a FFD technique for mesh deformation and an algebraic method [48] for the displacement of a high-stretching grid.

\section{Conclusions}

In this work, the continuous adjoint Spalart-Allmaras approach to aerodynamic design optimization has been presented. The SpalartAllmaras model has been chosen in order to describe turbulence phenomena due to its wide use in industrial applications of aerodynamics. However, the framework presented here for the turbulent continuous adjoint approach is general, and it can be easily extended to incorporate other models of turbulence, such as $k-\epsilon, k-\omega$, or shear stress models. The resulting formulation does not need the computation of second-order derivatives of the flow variables, and once the adjoint variables have been computed, it only requires an integration on the surface of the aerodynamical body in order to evaluate the gradient of the functional of interest under deformations of the body surface.

The accuracy of the sensitivity derivatives that result from the application of the new method has been assessed by comparison with finite difference computations, which illustrate the importance of the complete formulation instead of the frozen viscosity strategy. This improvement in the quality of the computed gradients is also clearly translated into the optimization process, with the complete turbulent adjoint being able to produce better optimized profiles than those obtained using frozen viscosity. Finally, a 3-D test case, making use of a combination of the continuous adjoint approach with advanced freeform techniques for mesh deformation, has also been presented, thus highlighting the potential flexibility of the continuous adjoint approach in realistic 3-D industrial applications.

The results presented here are very promising, but further numerical tests are still necessary in order to face the industrialization of the complete continuous adjoint methodology. In particular, the optimization of full complex 3-D configurations and convergence improvements are open research topics that have to be addressed.

\section{Appendix A. Navier-Stokes Equations}

As usual in the Navier-Stokes equations, system equation (1) separately considers the convective terms, denoted by $\mathbf{F}^{c}$, and the viscous ones, denoted here by $\mathbf{F}^{v 1}$ and $\mathbf{F}^{v 2}$. They are given by

$$
\begin{gathered}
\mathbf{F}_{i}^{c}=\left(\begin{array}{c}
\rho v_{i} \\
\rho v_{i} v_{1}+P \delta_{i 1} \\
\rho v_{i} v_{2}+P \delta_{i 2} \\
\rho v_{i} v_{3}+P \delta_{i 3} \\
\rho v_{i} H
\end{array}\right), \quad \mathbf{F}_{i}^{v 1}=\left(\begin{array}{c}
\cdot \\
\tau_{i 1} \\
\tau_{i 2} \\
\tau_{i 3} \\
v_{j} \tau_{i j}
\end{array}\right) \\
\mathbf{F}_{i}^{v 2}=\left(\begin{array}{c}
\cdot \\
\cdot \\
\cdot \\
\cdot \\
C_{p} \partial_{i} T
\end{array}\right), \quad i=1, \ldots, 3
\end{gathered}
$$

where $v_{i}$ are the Cartesian velocity components, $H$ is the fluid enthalpy, $\delta_{i j}$ is the Kronecker delta function, and $\tau_{i j}=\partial_{j} v_{i}+$ $\partial_{i} v_{j}-\frac{2}{3} \delta_{i j} \nabla \cdot \mathbf{v}$. Recall that Latin indexes $i, j$ denote 3-D Cartesian coordinates, with repeated indexes implying summation. In these formulas, $C_{p}$ is the specific heat at constant pressure, $T=P / R \rho$ is the temperature, and $R$ is the gas constant, so that, for an ideal gas, $C_{p} / R=\gamma /(\gamma-1)$, with $\gamma$ constant. To close the system, the 
dynamic viscosity is assumed to satisfy Sutherland's law, $\mu_{\mathrm{dyn}}=\mu_{1} T^{3 / 2} /\left(T+\mu_{2}\right)$, where $\mu_{1}$ and $\mu_{2}$ are also specified constants.

\section{Appendix B. Spalart-Allmaras Turbulence Model}

In Eq. (2), we have considered a general framework for a oneequation turbulence model. Here, we focus on the Spalart-Allmaras model, for which explicit formulas for the adjoint formulation are obtained below. In this case, the turbulent viscosity is computed as

$$
\mu_{\mathrm{tur}}=\rho \hat{v} f_{v 1}, \quad f_{v 1}=\frac{\chi^{3}}{\chi^{3}+c_{v 1}^{3}}, \quad \chi=\frac{\hat{v}}{v}, \quad v=\frac{\mu_{\mathrm{dyn}}}{\rho}
$$

The new variable $\hat{v}$ is obtained by solving Eq. (2) with

$$
\begin{gathered}
\mathbf{T}^{c v}(U, \hat{v})=-\frac{v+\hat{v}}{\sigma} \nabla \hat{v}+\mathbf{v} \hat{v} \\
T^{s}\left(U, \hat{v}, d_{S}\right)=c_{b 1} \hat{S} \hat{v}-c_{w 1} f_{w}\left(\frac{\hat{v}}{d_{S}}\right)^{2}+\frac{c_{b 2}}{\sigma}|\nabla \hat{v}|^{2}
\end{gathered}
$$

where the production term $\hat{S}$ in Eq. (B3) is defined as $\hat{S}=|\boldsymbol{\omega}|+\left(\hat{v} / \kappa^{2} d_{S}^{2}\right) f_{v 2}, \boldsymbol{\omega}=\nabla \times \mathbf{v}$ is the fluid vorticity, $d_{S}$ is the distance to the nearest wall, and $f_{v 2}=1-\left[\chi /\left(1+\chi f_{v 1}\right)\right]$. The function $f_{w}$ in Eq. (B3) is computed as $f_{w}=g\left[\left(1+c_{w 3}^{6}\right) /\right.$ $\left.\left(g^{6}+c_{w 3}^{6}\right)\right]^{1 / 6}$, where $g=r+c_{w 2}\left(r^{6}-r\right)$ and $r=\hat{v} / \hat{S} \kappa^{2} d_{S}^{2}$.

Finally, the set of closure constants for the model is given by $\sigma=2 / 3, c_{b 1}=0.1355, c_{b 2}=0.622, \kappa=0.41, c_{w 1}=\left(c_{b 1} / \kappa^{2}\right)+$ $\left[\left(1+c_{b 2}\right) / \sigma\right], c_{w 2}=0.3, c_{w 3}=2$, and $c_{v 1}=7.1$

\section{Appendix C. Linearized Navier-Stokes Equations}

In this appendix, we compute $\left(\partial R_{U} / \partial U\right) \delta U$ and $\left(\partial R_{U} / \partial \hat{v}\right) \delta \hat{v}$ in Eq. (14):

$$
\begin{gathered}
\frac{\partial R_{U}}{\partial U} \delta U=\nabla\left(\mathbf{A}^{c} \delta U\right)-\nabla \cdot\left(\mathbf{F}^{v k} \frac{\partial \mu_{\mathrm{tot}}^{k}}{\partial U} \delta U+\mu_{\mathrm{tot}}^{k} \mathbf{A}^{v k} \delta U\right. \\
\left.+\mu_{\mathrm{tot}}^{k} \mathbf{D}^{v k} \nabla \delta U\right) \\
\frac{\partial R_{U}}{\partial \hat{v}} \delta \hat{v}=-\nabla \cdot\left(\mathbf{F}^{v k} \frac{\partial \mu_{\mathrm{tot}}^{k}}{\partial \hat{v}} \delta \hat{v}\right)
\end{gathered}
$$

where

$$
\begin{aligned}
& \frac{\partial \mu_{\mathrm{tot}}^{1}}{\partial U}=\frac{\partial \mu_{\mathrm{dyn}}}{\partial U}+\frac{\partial \mu_{\mathrm{tur}}}{\partial U}, \quad \frac{\partial \mu_{\mathrm{tot}}^{2}}{\partial U}=\frac{1}{P r_{d}} \frac{\partial \mu_{\mathrm{dyn}}}{\partial U}+\frac{1}{P r_{t}} \frac{\partial \mu_{\mathrm{tur}}}{\partial U} \\
& \frac{\partial \mu_{\mathrm{tot}}^{1}}{\partial \hat{v}}=\frac{\partial \mu_{\mathrm{tur}}}{\partial \hat{v}}, \quad \frac{\partial \mu_{\mathrm{tot}}^{2}}{\partial \hat{v}}=\frac{1}{P r_{t}} \frac{\partial \mu_{\mathrm{tur}}}{\partial \hat{v}}
\end{aligned}
$$

and

$$
\frac{\partial \mu_{\mathrm{dyn}}}{\partial U}=\frac{\partial \mu_{\mathrm{dyn}}}{\partial T} \frac{\partial T}{\partial U}, \quad \frac{\partial \mu_{\mathrm{dyn}}}{\partial T}=\mu_{\mathrm{dyn}} \frac{T+3 \mu_{2}}{2 T\left(T+\mu_{2}\right)}
$$

$$
\frac{\partial \mu_{\mathrm{tur}}}{\partial U}=\hat{v} f_{v 1}\left(1+\frac{3 c_{v 1}^{3}}{\chi^{3}+c_{v 1}^{3}}\right) \frac{\partial \rho}{\partial U}-\chi f_{v 1} \frac{3 c_{v 1}^{3}}{\chi^{3}+c_{v 1}^{3}} \frac{\partial \mu_{\mathrm{dyn}}}{\partial U}
$$

$$
\frac{\partial \mu_{\mathrm{tur}}}{\partial \hat{v}}=\rho f_{v 1}\left(1+\frac{3 c_{v 1}^{3}}{\chi^{3}+c_{v 1}^{3}}\right)
$$

with

$$
\frac{\partial T}{\partial U}=\frac{(\gamma-1)}{R \rho}\left(|\mathbf{v}|^{2}-E,-v_{1},-v_{2},-v_{3}, 1\right)
$$

and $\partial \rho / \partial U=(1,0,0,0,0)$.

In Eq. (C1), we have

$$
\left.\begin{array}{cc}
\mathbf{A}^{c}=\left(A_{x}^{c}, A_{y}^{c}, A_{z}^{c}\right), & A_{i}^{c}=\left.\frac{\partial \mathbf{F}_{i}^{c}}{\partial U}\right|_{U(x, y, z)} \\
\mathbf{A}^{v k}=\left(A_{x}^{v k}, A_{y}^{v k}, A_{z}^{v k}\right), & A_{i}^{v k}=\left.\frac{\partial \mathbf{F}_{i}^{v k}}{\partial U}\right|_{U(x, y, z)} \\
\mathbf{D}^{v k}=\left(\begin{array}{ccc}
D_{x x}^{v k} & D_{x y}^{v k} & D_{x z}^{v k} \\
D_{y x}^{v k} & D_{y y}^{v k} & D_{y z}^{v k} \\
D_{z x}^{v k} & D_{z y}^{v k} & D_{z z}^{v k}
\end{array}\right), & D_{i j}^{v k}=\left.\frac{\partial \mathbf{F}_{i}^{v k}}{\partial\left(\partial_{j} U\right)}\right|_{U(x, y, z)}
\end{array}\right\}
$$

Defining for convenience, $a_{0}=(\gamma-1)$ and $\phi=(\gamma-1)\left(|\mathbf{v}|^{2} / 2\right)$; then, we have

$$
\begin{aligned}
& A_{i}^{c}=\left(\begin{array}{ccccc}
\cdot & \delta_{i 1} & \delta_{i 2} & \delta_{i 3} & \cdot \\
-v_{i} v_{1}+\delta_{i 1} \phi & v_{i}-\left(a_{0}-1\right) v_{i} \delta_{i 1} & v_{1} \delta_{i 2}-a_{0} v_{2} \delta_{i 1} & v_{1} \delta_{i 3}-a_{0} v_{3} \delta_{i 1} & a_{0} \delta_{i 1} \\
-v_{i} v_{2}+\delta_{i 2} \phi & v_{2} \delta_{i 1}-a_{0} v_{1} \delta_{i 2} & v_{i}-\left(a_{0}-1\right) v_{i} \delta_{i 2} & v_{2} \delta_{i 3}-a_{0} v_{3} \delta_{i 2} & a_{0} \delta_{i 2} \\
-v_{i} v_{3}+\delta_{i 3} \phi & v_{3} \delta_{i 1}-a_{0} v_{1} \delta_{i 3} & v_{3} \delta_{i 2}-a_{0} v_{2} \delta_{i 3} & v_{i}-\left(a_{0}-1\right) v_{i} \delta_{i 3} & a_{0} \delta_{i 3} \\
v_{i}(\phi-H) & -a_{0} v_{i} v_{1}+H \delta_{i 1} & -a_{0} v_{i} v_{2}+H \delta_{i 2} & -a_{0} v_{i} v_{3}+H \delta_{i 3} & \gamma v_{i}
\end{array}\right) \\
& A_{i}^{v 1}=\left(\begin{array}{ccccc}
\cdot & \partial_{i}\left(\frac{1}{\rho}\right)+\frac{1}{3} \partial_{1}\left(\frac{1}{\rho}\right) \delta_{i 1} & \partial_{1}\left(\frac{1}{\rho}\right) \delta_{i 2}-\frac{2}{3} \partial_{2}\left(\frac{1}{\rho}\right) \delta_{i 1} & \partial_{1}\left(\frac{1}{\rho}\right) \delta_{i 3}-\frac{2}{3} \partial_{3}\left(\frac{1}{\rho}\right) \delta_{i 1} & . \\
-\eta_{i 2} & \partial_{2}\left(\frac{1}{\rho}\right) \delta_{i 1}-\frac{2}{3} \partial_{1}\left(\frac{1}{\rho}\right) \delta_{i 2} & \partial_{i}\left(\frac{1}{\rho}\right)+\frac{1}{3} \partial_{2}\left(\frac{1}{\rho}\right) \delta_{i 2} & \partial_{2}\left(\frac{1}{\rho}\right) \delta_{i 3}-\frac{2}{3} \partial_{3}\left(\frac{1}{\rho}\right) \delta_{i 2} & . \\
-\eta_{i 3} & \partial_{3}\left(\frac{1}{\rho}\right) \delta_{i 1}-\frac{2}{3} \partial_{1}\left(\frac{1}{\rho}\right) \delta_{i 3} & \partial_{3}\left(\frac{1}{\rho}\right) \delta_{i 2}-\frac{2}{3} \partial_{2}\left(\frac{1}{\rho}\right) \delta_{i 3} & \partial_{i}\left(\frac{1}{\rho}\right)+\frac{1}{3} \partial_{3}\left(\frac{1}{\rho}\right) \delta_{i 3} & . \\
v_{j} \pi_{i j} & v_{j} \partial_{j}\left(\frac{1}{\rho}\right) \delta_{i 1}+\zeta_{i 1}+\frac{1}{\rho} \tau_{i 1} & v_{j} \partial_{j}\left(\frac{1}{\rho}\right) \delta_{i 2}+\zeta_{i 2}+\frac{1}{\rho} \tau_{i 2} & v_{j} \partial_{j}\left(\frac{1}{\rho}\right) \delta_{i 3}+\zeta_{i 3}+\frac{1}{\rho} \tau_{i 3} & .
\end{array}\right)
\end{aligned}
$$

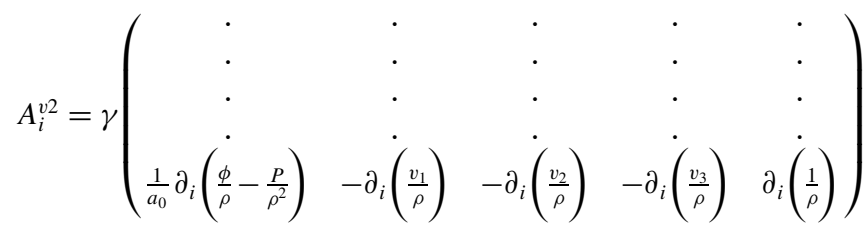




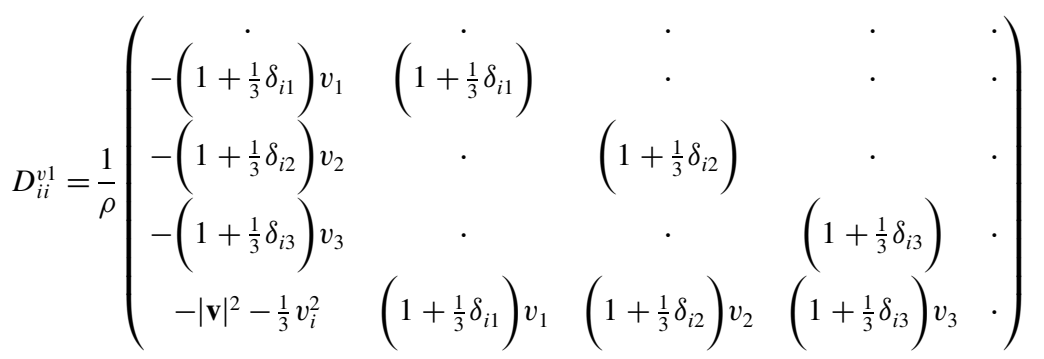

$$
\begin{aligned}
& D_{i j}^{v 1}=\frac{1}{\rho}\left(\begin{array}{ccccc}
-v_{i} \delta_{j 1}+\frac{2}{3} v_{j} \delta_{i 1} & \delta_{j 1} \delta_{i 1}-\frac{2}{3} \delta_{i 1} \delta_{j 1} & \delta_{j 1} \delta_{i 2}-\frac{2}{3} \delta_{i 1} \delta_{j 2} & \delta_{j 1} \delta_{i 3}-\frac{2}{3} \delta_{i 1} \delta_{j 3} & \cdot \\
-v_{i} \delta_{j 2}+\frac{2}{3} v_{j} \delta_{i 2} & \delta_{j 2} \delta_{i 1}-\frac{2}{3} \delta_{i 2} \delta_{j 1} & \delta_{j 2} \delta_{i 2}-\frac{2}{3} \delta_{i 2} \delta_{j 2} & \delta_{j 2} \delta_{i 3}-\frac{2}{3} \delta_{i 2} \delta_{j 3} & \cdot \\
-v_{i} \delta_{j 3}+\frac{2}{3} v_{j} \delta_{i 3} & \delta_{j 3} \delta_{i 1}-\frac{2}{3} \delta_{i 3} \delta_{j 1} & \delta_{j 3} \delta_{i 2}-\frac{2}{3} \delta_{i 3} \delta_{j 2} & \delta_{j 3} \delta_{i 3}-\frac{2}{3} \delta_{i 3} \delta_{j 3} & \cdot \\
-\frac{1}{3} v_{i} v_{j} & v_{j} \delta_{i 1}-\frac{2}{3} v_{i} \delta_{j 1} & v_{j} \delta_{i 2}-\frac{2}{3} v_{i} \delta_{j 2} & v_{j} \delta_{i 3}-\frac{2}{3} v_{i} \delta_{j 3} & \cdot
\end{array}\right) \quad(i \neq j) \\
& D_{i i}^{v 2}=\frac{\gamma}{\rho}\left(\begin{array}{ccccc}
\cdot & \cdot & \cdot & \cdot & \cdot \\
\cdot & \cdot & \cdot & \cdot & \cdot \\
\cdot & \cdot & \cdot & \cdot & \cdot \\
\cdot & \cdot & \cdot & \cdot & \cdot \\
\frac{1}{a_{0}}\left(\phi-\frac{P}{\rho}\right) & -v_{1} & -v_{2} & -v_{3} & 1
\end{array}\right) \quad D_{i j}^{\nu 2}=\mathbf{0}_{5 \times 5} \quad(i \neq j)
\end{aligned}
$$

where tensors $\bar{\eta}, \bar{\pi}$, and $\bar{\zeta}$ in the definition of $A_{i}^{v 1}$ are given by

$$
\begin{aligned}
\eta_{i j} & =\partial_{i}\left(\frac{v_{j}}{\rho}\right)+\partial_{j}\left(\frac{v_{i}}{\rho}\right)-\frac{2}{3} \delta_{i j} \nabla \cdot\left(\frac{\mathbf{v}}{\rho}\right) \\
\pi_{i j} & =v_{j} \partial_{i}\left(\frac{1}{\rho}\right)+v_{i} \partial_{j}\left(\frac{1}{\rho}\right)-\frac{2}{3} \delta_{i j} \mathbf{v} \cdot \nabla\left(\frac{1}{\rho}\right)=\eta_{i j}-\frac{1}{\rho} \tau_{i j} \\
\zeta_{i j} & =v_{j} \partial_{i}\left(\frac{1}{\rho}\right)-v_{i} \partial_{j}\left(\frac{1}{\rho}\right)+\frac{1}{3} v_{i} \partial_{j}\left(\frac{1}{\rho}\right)
\end{aligned}
$$

\section{Appendix D. Linearized Spalart-Allmaras}

\section{Turbulence Model}

Here, we compute the terms corresponding to linearized turbulence equation (15). Note that

$$
\begin{gathered}
\frac{\partial R_{\hat{v}}}{\partial U} \delta U=\nabla \cdot\left(\mathbf{F}^{c v} \delta U\right)-F^{s} \delta U-\mathbf{M}^{s} \nabla \delta U \\
\frac{\partial R_{\hat{v}}}{\partial \hat{v}} \delta \hat{v}=\nabla \cdot\left(\mathbf{B}^{c v} \delta \hat{v}+\mathbf{E}^{c v} \nabla \delta \hat{v}\right)-B^{s} \delta \hat{v}-\mathbf{E}^{s} \nabla \delta \hat{v} \\
\frac{\partial R_{\hat{v}}}{\partial d_{S}} \delta d_{S}=-K^{s} \delta d_{S}
\end{gathered}
$$

where $\quad \mathbf{M}^{s}=\left(M_{x}^{s}, M_{y}^{s}, M_{z}^{s}\right), \quad \mathbf{E}^{c v}=\left(E_{x}^{c v}, E_{y}^{c v}, E_{z}^{c v}\right), \quad$ and $\mathbf{E}^{s}=\left(E_{x}^{s}, E_{y}^{s}, E_{z}^{s}\right)$.

The Jacobian matrices associated to the convective/viscous flux are given by

$$
\begin{gathered}
\mathbf{B}^{c v}=\frac{\partial \mathbf{T}^{c v}}{\partial \hat{v}}=-\frac{\nabla \hat{v}}{\sigma}+\mathbf{v} \\
E_{i}^{c v}=\frac{\partial \mathbf{T}^{c v}}{\partial\left(\partial_{i} \hat{v}\right)}=-\frac{v+\hat{v}}{\sigma} \\
\mathbf{F}^{c v}=\frac{\partial \mathbf{T}^{c v}}{\partial U}=\alpha\left(\frac{\partial T}{\partial U}\right)^{\top} \nabla \hat{v}+\left(\begin{array}{c}
\frac{\mu_{\mathrm{dyn}}}{\sigma \rho^{2}} \nabla \hat{v}-\frac{\hat{v}}{\rho} \mathbf{v} \\
\frac{\hat{v}}{\rho} \mathbf{I}_{3} \\
\cdot
\end{array}\right), \\
\alpha=-\frac{1}{\sigma \rho} \frac{\partial \mu_{\mathrm{dyn}}}{\partial T}
\end{gathered}
$$

Here, $\partial \mu_{\mathrm{dyn}} / \partial T$ and $\partial T / \partial U$ are given in Appendix $\underline{\mathbf{C}}$, and $\mathbf{I}_{3}$ is the $3 \times 3$ identity matrix.

Concerning the derivatives of the source term $T^{s}$, we have

$$
\begin{aligned}
B^{s} & =\frac{\partial T^{s}}{\partial \hat{v}}=\left(c_{b 1} \hat{S}-2 c_{w 1} f_{w} \frac{\hat{v}}{d_{S}^{2}}\right)-c_{w 1}\left[\frac{\hat{v}}{d_{S}}\right]^{2} f_{w}^{g} g^{r} r^{\hat{v}} \\
& +\Lambda\left(\hat{S}^{f_{v 2}}\left(f_{v 2}^{\chi}+f_{v 2}^{v 1} f_{v 1}^{\chi}\right) \chi^{\hat{v}}+\hat{S}^{\hat{v}}\right) \\
F^{s} & =\frac{\partial T^{s}}{\partial U}=\Lambda\left[\hat{S}^{f_{v 2}}\left(f_{v 2}^{\chi}+f_{v 2}^{v 1} f_{v 1}^{\chi}\right) \chi^{v}\left(v^{T} \frac{\partial T}{\partial U}+v^{\rho} \frac{\partial \rho}{\partial U}\right)^{\top}\right. \\
+ & \left.\frac{1}{|\omega|}\left(\partial_{i} \mathbf{v}-\nabla v_{i}\right) \cdot \partial_{i} N\right]
\end{aligned}
$$

$$
K^{s}=\frac{\partial T^{s}}{\partial d_{S}}=\frac{2 c_{w 1} f_{w} \hat{v}^{2}}{d_{S}^{3}}+\Lambda \hat{S}^{d_{S}}
$$

$$
E_{i}^{s}=\frac{\partial T^{s}}{\partial\left(\partial_{i} \hat{v}\right)}=2 \frac{c_{b 2}}{\sigma} \partial_{i} \hat{v}
$$

$$
M_{i}^{s}=\frac{\partial T^{s}}{\partial\left(\partial_{i} U\right)}=\frac{1}{|\omega|} \Lambda\left(\partial_{i} \mathbf{v}-\nabla v_{i}\right) \cdot N
$$

with

$$
\Lambda=\frac{\partial T^{s}}{\partial \hat{S}}=c_{b 1} \hat{v}-c_{w 1}\left[\frac{\hat{v}}{d_{S}}\right]^{2} f_{w}^{g} g^{r} r^{\hat{S}}
$$

and where $N$ in Eqs. (D8) and (D11) is the $3 \times 5$ matrix defined by $\delta \mathbf{v}=N \delta U$; that is,

$$
N=\frac{1}{\rho}\left(\begin{array}{ccccc}
-v_{1} & 1 & \cdot & \cdot & \cdot \\
-v_{2} & \cdot & 1 & \cdot & \cdot \\
-v_{3} & \cdot & \cdot & 1 & \cdot
\end{array}\right)
$$

Finally, the whole set of partial derivatives needed to compute Eqs. (D7-D12) reads 


$$
\begin{aligned}
& f_{w}^{g}=\frac{\partial f_{w}}{\partial g}=\frac{c_{w 3}^{6}}{g^{6}+c_{w 3}^{6}}\left(\frac{1+c_{w 3}^{6}}{g^{6}+c_{w 3}^{6}}\right)^{1 / 6} \\
& g^{r}=\frac{\partial g}{\partial r}=1+c_{w 2}\left(6 r^{5}-1\right) \quad r^{\hat{v}}=\frac{\partial r}{\partial \hat{v}}=\frac{1}{\hat{S} \kappa^{2} d_{S}^{2}} \\
& r^{\hat{S}}=\frac{\partial r}{\partial \hat{S}}=-\frac{\hat{v}}{\hat{S}^{2} \kappa^{2} d_{S}^{2}} \quad r^{d_{S}}=\frac{\partial r}{\partial d_{S}}=-\frac{2 \hat{v}}{\hat{S} \kappa^{2} d_{S}^{3}} \\
& \hat{S}^{f_{v 2}}=\frac{\partial \hat{S}}{\partial f_{v 2}}=\frac{\hat{v}}{\kappa^{2} d_{S}^{2}} \quad \hat{S}^{\hat{v}}=\frac{\partial \hat{S}}{\partial \hat{v}}=\frac{f_{v 2}}{\kappa^{2} d_{S}^{2}} \\
& \hat{S}^{d_{S}}=\frac{\partial \hat{S}}{\partial d_{S}}=-\frac{2 \hat{v} f_{v 2}}{\kappa^{2} d_{S}^{3}} \quad f_{v 2}^{\chi}=\frac{\partial f_{v 2}}{\partial \chi}=-\frac{1}{\left(1+\chi f_{v 1}\right)^{2}} \\
& f_{v 2}^{v 1}=\frac{\partial f_{v 2}}{\partial f_{v 1}}=\frac{\chi^{2}}{\left(1+\chi f_{v 1}\right)^{2}} \quad f_{v 1}^{\chi}=\frac{\partial f_{v 1}}{\partial \chi}=\frac{3 \chi^{2} c_{v 1}^{3}}{\left(\chi^{3}+c_{v 1}^{3}\right)^{2}} \\
& \chi^{\hat{v}}=\frac{\partial \chi}{\partial \hat{v}}=\frac{1}{v} \quad \chi^{v}=\frac{\partial \chi}{\partial \nu}=-\frac{\chi}{v} \quad v^{\rho}=\frac{\partial v}{\partial \rho}=-\frac{\mu_{\mathrm{dyn}}}{\rho^{2}} \\
& v^{T}=\frac{\partial v}{\partial T}=\frac{1}{\rho} \frac{\partial \mu_{\mathrm{dyn}}}{\partial T} \quad{ }^{2}
\end{aligned}
$$

\section{Appendix E. Adjoint Formulas}

In this appendix, we give explicit formulas for the adjoint operators and boundary conditions. These are obtained from identity equation (17), which is deduced from the following integration by parts:

$$
\begin{gathered}
\int_{\Omega} \Psi_{U}^{\top}\left(\frac{\partial R_{U}}{\partial U} \delta U+\frac{\partial R_{U}}{\partial \hat{v}} \delta \hat{v}\right)=\int_{\Omega}\left(A_{U}^{U} \Psi_{U}\right)^{\top} \delta U+\int_{\Omega} A_{U}^{\hat{v}} \Psi_{U} \delta \hat{v} \\
-\int_{S} \boldsymbol{\varphi} \cdot(\delta P \mathbf{n}-\delta \bar{\sigma} \cdot \mathbf{n})-\int_{S}\left(\mathbf{g}_{1} \cdot \boldsymbol{\varphi}+g_{2} \partial_{n} \psi_{5}\right) \delta T-\int_{S} h \delta S
\end{gathered}
$$

$$
\begin{aligned}
& \int_{\Omega} \psi_{\hat{v}}\left(\frac{\partial R_{\hat{v}}}{\partial U} \delta U+\frac{\partial R_{\hat{v}}}{\partial \hat{v}} \delta \hat{v}+\frac{\partial R_{\hat{v}}}{\partial d_{S}} \delta d_{S}\right)=\int_{\Omega} A_{\hat{v}}^{U} \psi_{\hat{v}} \delta U \\
& +\int_{\Omega} A_{\hat{v}}^{\hat{v}} \psi_{\hat{v}} \delta \hat{v}+\int_{\Omega} A_{\hat{v}}^{d} \psi_{d} \delta \hat{v}-\int_{S} \psi_{\hat{v}} g_{3} \partial_{n}(\delta \hat{v})+\int_{S} \psi_{\hat{v}} g_{4} \delta P \\
& \quad-\int_{S} \psi_{\hat{v}} g_{5} \delta T+\int_{S} \frac{1+2 c_{b 2}}{\sigma}\left(\partial_{n} \hat{v}\right)^{2} \psi_{\hat{v}} \delta S
\end{aligned}
$$$$
\int_{\Omega} \psi_{d} \nabla d_{S} \cdot \nabla \delta d_{S}=\int_{\Omega} A_{d}^{d} \delta d_{S}-\int_{S} \psi_{d} \delta S
$$

where we have used $\partial_{n} d_{S}=-1$. Here, domain integrals on the righthand side contain the adjoint operators, given by

$$
\begin{gathered}
A_{U}^{U} \Psi_{U}=-\nabla \Psi_{U}^{\top} \cdot \mathbf{A}^{c}-\nabla \cdot\left(\nabla \Psi_{U}^{\top} \cdot \mu_{\mathrm{tot}}^{k} \mathbf{D}^{v k}\right) \\
+\nabla \Psi_{U}^{\top} \cdot \mu_{\mathrm{tot}}^{k} \mathbf{A}^{v k}+\nabla \Psi_{U}^{\top} \cdot \mathbf{F}^{v k} \frac{\partial \mu_{\mathrm{tot}}^{k}}{\partial U} \\
A_{\hat{v}}^{U} \psi_{\hat{v}}=-\nabla \psi_{\tilde{v}} \cdot \mathbf{F}^{c v}-\psi_{\hat{v}} F^{s}+\nabla \cdot\left(\psi_{\hat{v}} \mathbf{M}^{s}\right) \\
A_{U}^{\hat{v}} \Psi_{U}=\nabla \Psi_{U}^{\top} \cdot \mathbf{F}^{v k} \frac{\partial \mu_{\mathrm{tot}}^{k}}{\partial \hat{v}}
\end{gathered}
$$

$$
A_{\hat{v}}^{\hat{v}} \psi_{\hat{v}}=-\nabla \psi_{\hat{v}} \cdot \mathbf{B}^{c v}+\nabla \cdot\left(\nabla \psi_{\hat{v}} \cdot \mathbf{E}^{c v}\right)-\psi_{\hat{v}} B^{s}+\nabla \cdot\left(\psi_{\hat{v}} \mathbf{E}^{s}\right)
$$

$$
A_{\hat{v}}^{d} \psi_{\hat{v}}=-K^{s} \psi_{\hat{v}}
$$

$$
A_{d}^{d} \psi_{d}=-\nabla \cdot\left(\psi_{d} \nabla d_{S}\right)
$$

The terms $\mathbf{g}_{1}, g_{2}, g_{3}, g_{4}, g_{5}$, and $h$ appearing in the boundary integrals in Eqs. (E1-E3) are given by

$$
\begin{gathered}
\mathbf{g}_{1}=\frac{\partial \mu_{\mathrm{dyn}}}{\partial T} \mathbf{n} \cdot \bar{\tau}, \quad g_{2}=C_{p} \mu_{\mathrm{tot}}^{2}, \quad g_{3}=\frac{v}{\sigma} \\
g_{4}=\partial_{n} \hat{v} \frac{\mu_{\mathrm{dyn}}}{\sigma \rho P}, \quad g_{5}=\partial_{n} \hat{v} \frac{1}{\sigma \rho}\left(\frac{\partial \mu_{\mathrm{dyn}}}{\partial T}+\frac{\mu_{\mathrm{dyn}}}{T}\right) \\
h=-\left(\rho \psi_{1}+\rho H \psi_{5}\right)\left(\partial_{n} \mathbf{v} \cdot \mathbf{n}\right)+\psi_{5} \mathbf{n} \cdot \bar{\sigma} \cdot \partial_{n} \mathbf{v}-\mathbf{n} \cdot \bar{\Sigma}^{\varphi} \cdot \partial_{n} \mathbf{v} \\
-\psi_{5} \bar{\sigma}: \nabla \mathbf{v}+\mu_{\mathrm{tot}}^{2} C_{p} \nabla_{S} \psi_{5} \cdot \nabla_{S} T+\partial_{n} \hat{v}(\mathbf{n} \cdot \bar{\tau} \cdot \boldsymbol{\varphi}) \frac{\partial \mu_{\mathrm{tur}}}{\partial \hat{v}}
\end{gathered}
$$

where $\bar{\sigma}: \nabla \mathbf{v}=\sigma_{i j} \partial_{i} v_{j}$, with

$$
\bar{\Sigma}^{\varphi}=\mu_{\mathrm{tot}}^{1}\left(\nabla \boldsymbol{\varphi}+\nabla \boldsymbol{\varphi}^{\top}-\mathbf{I}_{d} \frac{2}{3} \nabla \cdot \boldsymbol{\varphi}\right)
$$

Some of the terms in $h$ above can be simplified. In particular, taking into account that $\mathbf{v}=0$ and $\nabla_{S} \mathbf{v}=0$ on the obstacle surface, we have $\nabla \mathbf{v}=\partial_{j} v_{i}=\partial_{n} v_{i} n_{j}$. Therefore,

$$
\bar{\sigma}: \nabla \mathbf{v}=\sigma_{i j} \partial_{n} v_{i} n_{j}=\mathbf{n} \cdot \bar{\sigma} \cdot \partial_{n} \mathbf{v} \quad \text { on } S
$$

and the second and fourth terms in Eq. (E11) cancel.

On the other hand, the continuity equation yields $\nabla \cdot \mathbf{v}=0$ on $S$. Hence,

$$
0=\nabla \cdot \mathbf{v}=\partial_{i} v_{i}=\partial_{n} v_{i} n_{i}=\partial_{n} \mathbf{v} \cdot \mathbf{n} \quad \text { on } S
$$

and the first term in Eq. (E11) also cancels.

In the same way, one may also notice that $\partial \mu_{\text {tur }} / \partial \hat{v}=0$ on $S$, since $f_{v 1}=0$ on the obstacle surface. Thus, the term $h$ reads

$$
h=-\mathbf{n} \cdot \bar{\Sigma}^{\varphi} \cdot \partial_{n} \mathbf{v}+\mu_{\mathrm{tot}}^{2} C_{p} \nabla_{S} \psi_{5} \cdot \nabla_{S} T
$$

Finally, adding the three terms in Eqs. (E1-E3), we easily obtain Eq. (17) with

$$
\hat{g}=h-\frac{1+2 c_{b 2}}{\sigma}\left(\partial_{n} \hat{v}\right)^{2} \psi_{\hat{v}}
$$

\section{Acknowledgments}

The research described in this paper has been supported under the FuSim-E Programme, funded by Airbus Spain, and by grants MTM2008-03541 and MTM2011-29306 of the Ministerio de Ciencia e Innovación (Spain). Francisco Palacios is also supported by the U.S. Department of Energy under the Predictive Science Academic Alliance Program, and Enrique Zuazua is supported by project PI2010-04 of the Basque Government, the European Research Council advanced grant FP7-246775 New Analytical and Numerical Methods in Wave Propagation (NUMERIWAVES), and the European Science Foundation's Research Networking Program: Optimization with Partial Differential Equation Constraints (OPTPDE).

\section{References}

[1] Spalart, P., and Allmaras, S., "A One-Equation Turbulence Model for Aerodynamic Flows," AIAA Paper 1992-0439, 1992.

[2] Jameson, A., "Aerodynamic Design via Control Theory," Journal of Scientific Computing, Vol. 3, No. 3, 1988, pp. 233-260. doi:10.1007/BF01061285

[3] Pironneau, O., "On Optimum Design in Fluid Mechanics," Journal of Fluid Mechanics, Vol. 64, 1974, pp. 97-110. 
doi:10.1017/S0022112074002023

[4] Anderson, W. K., and Venkatakrishnan, V., "Aerodynamic Design Optimization on Unstructured Grids with a Continuous Adjoint Formulation," AIAA Paper 1997-0643, 1997.

[5] Castro, C., Lozano, C., Palacios, F., and Zuazua, E., "A Systematic Continuous Adjoint Approach to Viscous Aerodynamic Design on Unstructured Grids," AIAA Journal, Vol. 45, No. 9, 2007, pp. 2125-2139. doi: $10.2514 / 1.24859$

[6] Nadarajah, S. K., and Jameson, A., "A Comparison of the Continuous and Discrete Adjoint Approach to Automatic Aerodynamic Optimization," AIAA Paper 2000-0667, 2000.

[7] Giles, M. B., Discrete Adjoint Approximations with Shocks: Hyperbolic Problems: Theory, Numerics, Applications, Springer-Verlag, New York, 2003, pp. 185-194.

[8] Giles, M. B., and Ulbrich, S., "Convergence of Linearized and Adjoint Approximations for Discontinuous Solutions of Conservation Laws. Part 1: Linearized Approximations and Linearized Output Functionals," SIAM Journal on Numerical Analysis, Vol. 48, No. 3, 2010, pp. 882-904. doi: $10.1137 / 080727464$

[9] Giles, M. B., and Ulbrich, S., "Convergence of Linearized and Adjoint Approximations for Discontinuous Solutions of Conservation Laws. Part 2: Adjoint Approximations and Extensions," SIAM Journal on Numerical Analysis, Vol. 48, No. 3, 2010, pp. 905-921. doi:10.1137/09078078X

[10] Hartmann, R., "Adjoint Consistency Analysis of Discontinuous Galerkin Discretizations," SIAM Journal on Numerical Analysis, Vol. 45, No. 6, 2007 doi:10.1137/060665117

[11] Giles, M. B., and Pierce, N., "Analytic Adjoint Solutions for the QuasiOne-Dimensional Euler Equations," Journal of Fluid Mechanics, Vol. 426, 2001, pp. 327-345. doi:10.1017/S0022112000002366

[12] Martins, J. R. R. A., Sturdza, P., and Alonso, J. J., "The Complex-Step Derivative Approximation," ACM Transactions on Mathematical Software, Vol. 29, No. 3, 2003, pp. 245-262. doi: $10.1145 / 838250.838251$

[13] Mader, C., Martins, J. R. R. A., Alonso, J. J., and van der Weide, E., "ADjoint: An Approach for the Rapid Development of Discrete Adjoint Solvers," AIAA Journal, Vol. 46, No. 4, 2008, pp. 863-873. doi: $10.2514 / 1.29123$

[14] Dwight, R. P., and Brezillon, J., "Efficient and Robust Algorithms for Solution of the Adjoint Compressible Navier-Stokes Equations with Applications," International Journal for Numerical Methods in Fluids, Vol. 60, 2009, pp. 365-389. doi:10.1002/fld. 1894

[15] Peter, J. E. V., and Dwight, R. P., "Numerical Sensitivity Analysis for Aerodynamic Optimization: A Survey of Approaches," Computers and Fluids, Vol. 39, 2010, pp. 373-391. doi:10.1016/i.compfluid.2009.09.013

[16] Hicken, J. E., and Zingg, D. W., "Superconvergent Functional Estimates from Summation-by-Parts Finite-Difference Discretizations," SIAM Journal on Scientific Computing, Vol. 33, No. 2, 2011, pp. 893-922. doi: $10.1137 / 100790987$

[17] Zymaris, A. S., Papadimitriou, D. I., Giannakoglou, K. C., and Othmer, C., "Continuous Adjoint Approach to the Spalart-Allmaras Turbulence Model for Incompressible Flows," Computers and Fluids, Vol. 38, 2009 , pp. $1528-1538$.

doi:10.1016/j.compfluid.2008.12.006

[18] Nielsen, E., Lu, J., Park, M. A., and Darmofal, D. L., "An Implicit Exact Dual Adjoint Solution Method for Turbulent Flows on Unstructured Grids," Computers and Fluids, Vol. 33, 2004, pp. 1131-1155. doi:10.1016/j.compfluid.2003.09.005

[19] Dwight, R. P., and Brezillon, J., "Effect of Approximations of the Discrete Adjoint on Gradient-Based Optimization," AIAA Journal, Vol. 44, No. 12, 2006, pp. 3022-3031. doi: $10.2514 / 1.21744$

[20] Anderson, W. K., and Bonhaus, D. L., "Airfoil Design on Unstructured Grids for Turbulent Flows," AIAA Journal, Vol. 37, No. 2, 1999, pp. $185-191$ doi: $10.2514 / 2.712$

[21] Lee, B. J., and Kim, C., "Automated Design Methodology of Turbulent Internal Flow Using Discrete Adjoint Formulation," Aerospace Science and Technology, Vol. 11, 2007, pp. 163-173. doi:10.1016/j.ast.2006.12.001

[22] Mavriplis, D. J., "Discrete Adjoint-Based Approach for Optimization Problems on Three-Dimensional Unstructured Meshes," AIAA Journal,
Vol. 45, No. 4, 2007, pp. 740-750.

doi: $10.2514 / 1.22743$

[23] Kim, C. S., Kim, C., and Rho, O. H., "Feasibility Study of Constant Eddy-Viscosity Assumption in Gradient-Based Design Optimization," Journal of Aircraft, Vol. 40, No. 6, 2003, pp. 1168-1176. doi: $10.2514 / 2.7206$

[24] Deckelnick, K., Elliott, C. M., and Styles, V., "Optimal Control of the Propagation of a Graph in Inhomogeneous Media," SIAM Journal on Control and Optimization, Vol. 48, No. 3, 2009, pp. 13351352. doi: $10.1137 / 080723648$

[25] Deckelnick, K., Elliott, C. M., and Styles, V., "Numerical Analysis of an Inverse Problem for the Eikonal Equation," Numerische Mathematik, Vol. 119, No. 2, 2011, pp. 245-269. doi:10.1007/s00211-011-0386-z

[26] White, F., Viscous Fluid Flow, McGraw-Hill, New York, 1974.

[27] Landau, L. D., and Lifshitz, E. M., Fluid Mechanics, 2nd ed., Pergamon Press, New York, 1993.

[28] Wilcox, D., Turbulence Modeling for CFD, 2nd ed., DCW Industries, Inc., La Cañada, CA, 1998.

[29] Pironneau, O., Finite Element Methods for Fluids, Wiley, New York, 1990.

[30] Sokolowski, J., and Zolesio, J.-P., Introduction to Shape Optimization, Springer-Verlag, New York, 1991.

[31] Baeza, A., Castro, C., Palacios, F., and Zuazua, E., "2-D Euler Shape Design on Nonregular Flows Using Adjoint Rankine-Hugoniot Relations," AIAA Journal, Vol. 47, No. 3, 2009, pp. 552-562. doi:10.2514/1.37149

[32] Jameson, A., Schmidt, W., and Turkel, E., "Numerical Solution of the Euler Equations by Finite Volume Methods Using Runge-Kutta Time Stepping Schemes," AIAA Paper 1981-1259, 1981.

[33] Eliasson, P., "EDGE, a Navier-Stokes Solver for Unstructured Grids," FOI Swedish Defence Research Agency, TR FOI-R-0298-SE, Stockholm, 2002.

[34] Venkatakrishnan, V., "On the Accuracy of Limiters and Convergence to Steady State Solutions," AIAA Paper 1993-0880, 1993.

[35] Weiss, J. M., Maruszewski, J. P., and Wayne, A. S., "Implicit Solution of the Navier-Stokes Equation on Unstructured Meshes," AIAA Paper 1997-2103, 1997.

[36] Elias, R. N., Martins, M. A. D., and Coutinho, A. L. G. A., "Simple Finite Element-Based Computation of Distance Functions in Unstructured Grids," International Journal for Numerical Methods in Engineering, Vol. 72, No. 9, 2007, pp. 1095-1110. doi:10.1002/nme.2079

[37] Sethian, J. A., and Vladimirsky, A., "Fast Methods for the Eikonal and Related Hamilton-Jacobi Equations on Unstructured Meshes," Proceedings of the National Academy of Sciences of the United States of America, Vol. 97, No. 11, 2000, pp. 5699-5703. doi:10.1073/pnas.090060097

[38] Hirsch, C., Numerical Computation of Internal and External Flows, Wiley, New York, 1984, pp. 624-675.

[39] Jameson, A., and Turkel, E., "Implicit Schemes and LU Decompositions," Mathematics of Computation, Vol. 37, No. 156, 1981, pp. 385-397. doi:10.1090/S0025-5718-1981-0628702-9

[40] Jameson, A., Martinelli, L., and Grasso, F., "A Multigrid Method for the Navier-Stokes Equations," AIAA Paper 1986-0208, 1986.

[41] Jameson, A., Alonso, J. J., and Martinelli, L., "Multigrid Unsteady Navier-Stokes Calculations with Aeroelastic Applications," AIAA Paper 1995-0048, 1995.

[42] Hicks, R. M., and Henne, P. A., "Wing Design by Numerical Optimization," Journal of Aircraft, Vol. 15, 1978, pp. 407-412. doi: $10.2514 / 3.58379$

[43] Samareh, J. A., "Aerodynamic Shape Optimization Based on FreeForm Deformation," AIAA Paper 2004-4630, 2004.

[44] Degand, C., and Farhat, C., "A Three-Dimensional Torsional Spring Analogy Method for Unstructured Dynamic Meshes," Computers and Structures, Vol. 80, 2002, pp. 305-316. doi:10.1016/S0045-7949(02)00002-0

[45] Bottasso, C. L., Detomi, D., and Serra, R., "The Ball-Vertex Method: A New Simple Spring Analogy Method for Unstructured Dynamic Meshes," Computer Methods in Applied Mechanics and Engineering, Vol. 194, 2005, pp. 4244-4264 doi:10.1016/j.cma.2004.08.014

[46] Cook, P., McDonald, M., and Firmin, M., "Aerofoil RAE2822 Pressure Distributions, and Boundary Layer and Wake Measurements," AGARD TR 138, 1979.

[47] Haase, W., Bradsma, F., Elsholz, E., Leschziner, M., and Schwamborn, D. (eds.), EUROVAL: An European Initiative on Validation of CFD 
Codes, Vol. 42, Notes on Numerical Fluid Mechanics, Wiley, New York, 1993.

[48] Meaux, M., Cormery, M., and Voizard, G., "Viscous Aerodynamic Shape Optimization Based on the Discrete Adjoint State for 3-D Industrial Configurations," Proceedings of the European Congress on
Computational Methods in Applied Sciences (ECCOMAS), Paper 897. July 2004.

J. Martins Associate Editor 